\title{
Geometrically Thin Disk Accreting Into a Black Hole
}

\author{
N. Afshordi \& B. Paczyński \\ Princeton University Observatory, Princeton, NJ 08544-1001, USA \\ E-mail: afshordi@astro.princeton.edu \\ E-mail: bp@astro.princeton.edu
}

\begin{abstract}
A numerical model of a steady state, thin accretion disk with a constant effective speed of sound is presented. We demonstrate that 'zero torque' inner boundary condition is a reasonable approximation provided that the disk thickness, including the thickness of the torquing magnetic fields, is small everywhere. It is likely that this conclusion is correct also for non-steady disks, as long as the total thickness at the sonic point, $H_{c}$, is much smaller than the radius there, $r_{c} \approx r_{m s}$. The very existence of thin disks is not proved or disproved in this work, but such disks are believed to exist for moderate accretion rates. Within our model there is a small torque at $r_{m s}$, which may increase disk luminosity by several percent. An important result of our analysis is that the physically acceptable steady state solutions in our toy model exist only for $\alpha<0.14\left(100 v_{s} / c\right)^{1 / 3}$.

A significant torque may be applied to a thin disk if there is a large scale magnetic field, like in a modified Blandford-Znajek mechanism.
\end{abstract}

Subject headings: black hole physics — accretion disks — magnetic fields

\section{Introduction}

Theory of accretion disks is several decades old. With time ever more sophisticated and more diverse models of accretion onto black holes have been introduced. However, when it comes to modeling disk spectra, conventional steady state, geometrically thin disk models are still used, adopting the classical 'no torque' inner boundary condition at the marginally stable orbit at $r_{m s}$ (e.g. Blaes, Hubeny, Agol, \& Krolik 2001). Recently, the 'no torque' condition for geometrically thin disks has been challenged by several authors (Krolik 1999, Gammie 1999, Agol \& Krolik 2000, to be referred as AGK.) One of us (BP) did not agree with their claim, and presented simple arguments why the 'no torque' inner 
boundary condition is natural if an accretion disk is geometrically thin (Paczyński 2000), but the referee could not be convinced. Thanks to the electronic preprint server BP's paper is readily accessible to all interested readers, who can judge its validity.

In this paper we present a more detailed quantitative analysis of the inner boundary condition, refining arguments given by BP, and making them more precise. We find that AGK were qualitatively correct: a classical thin steady state disk must have some torque

at the $r_{m s}$, but the effect is not as strong as claimed by them. Following a short historical review of accretion disk theory in Section 2, we discuss the concept of thin disks, and we present equations that describe a disk with a fixed value of the effective speed of sound in Sec. 3. In Sec. 4 the solutions of these equations and their topologies are studied numerically. In Sec. 5 we describe an analytical method to identify solutions of different nature. In Sec. 6 we discuss some of the physical results of our work as well as those of Gammie (1999). In particular, we explain why the model and the reasoning proposed by Gammie, while bringing up an important issue, were quantitatively incorrect. Finally, Sec. 7 concludes this paper.

\section{Historical outline}

A Newtonian theory of geometrically thin accretion disks was developed in classical papers by Pringle \& Rees (1972), Shakura \& Sunyaev (1973) and Lynden-Bell \& Pringle (1974), generalized for a relativistic case by Novikov \& Thorne (1973), and reviewed by Pringle (1981). A relativistic effect: the bending of light trajectories leading to direct illumination of the disk by itself, was first elaborated and calculated by Cunningham (1975, 1976). This is marginally important for a disk accreting onto a Schwarzschild black hole, but it is important in the Kerr case, introducing significant non-local effects to the energy balance at small radii. In these early papers a geometrically thin disk accreting onto a black hole had the inner boundary at the marginally stable orbit, $r_{m s}$, and the gas was freely falling on a tight spiral inwards of $r_{m s}$.

There was some confusion about the nature of gas flow near the inner disk edge (Stoeger 1976). The issue was first understood as a transition from sub-sonic to supersonic flow for geometrically thick disks (Abramowicz et al. 1978), and later for geometrically thin disks (Muchotrzeb \& Paczyński 1982). Small $\alpha$-parameter used in these papers resulted in saddle-type critical points. Matsumoto et al. (1984) found that for moderately large values of $\alpha$ the critical point was nodal-type. These papers, and many more, were reviewed by Abramowicz \& Kato (1989), who presented a general discussion of the transonic flows in accretion disks. In particular, they pointed out that while such a transition is necessary 
for the existence of a steady state accretion, it does not guarantee that a sensible global solution exists (cf. their Fig. 2).

All the papers written in 1970s and 1980s adopted some form of 'alpha' viscosity, as there was no quantitative physical model for the effective transport of angular momentum and the dissipation of energy within accretion disks. Perhaps the best qualitative description of these processes was given by Galeyev et al. (1979). A major breakthrough was made by Balbus \& Hawley (1991) and Hawley \& Balbus (1991), who rediscovered a powerful magneto-rotational instability in weakly magnetized disks, and pointed out its relevance to accretion. A flood of papers followed, with powerful computers making it possible to model time dependent accretion flows in 2-D (Armitage 1998, Stone et al. 1999, Agol et al. 2001, Stone \& Pringle 2001, and references therein) and in 3-D (Flemming et al. 2000, Hawley et al. 2001, Armitage 2001, Armitage et al. 2001, Reynolds \& Armitage 2001, Reynolds et al. 2001, Sano \& Inutsuka 2001, Krolik \& Hawley 2002, Hawley \& Krolik 2002, Igumenshchev et al. 2003, and references therein).

A lot of semi-analytical and numerical work was done for the ADAF and CDAF models (cf. Ball, Narayan \& Quataert 2001, and references therein). It appears that at very high and at very low accretion rates disks are geometrically thick, while thin disks may exist only for moderate accretion rates. If this view is correct then the early work on geometrically thin accretion disks may be relevant for $0.01<L / L_{E d d}<0.1$.

A very interesting model of a geometrically thin disk with a geometrically thick magnetic corona was recently presented by Merloni (2003). Recent reviews of various modes of disk accretion onto black holes were provided by Merloni (2002) and by Blaes (2002).

Yet another way to modify the picture was proposed by Blandford and Znajek (1977), who pointed out that a large scale magnetic field may thread a black hole, and it may extract its rotational energy. In recent years the original picture was modified, making it more likely that the energy extracted from a spinning black hole is transferred to the inner parts of the disk, rather than directly to a distant load (Agol \& Krolik 2000, Li 2000a,b, Wang et al. 2002, and references therein). Recent XMM observations of an AGN named MCG-6-30-15 were claimed to support this possibility (Wilms et al. 2001, Merloni \& Fabian 2003). Cao \& Xu (2002) investigated local structure of the accretion flow near the sonic point assuming there is a steady torque exerted at the inner disk edge. 


\section{A Thin Disk}

The modern 2-D and 3-D numerical simulations provided the first quantitative and meaningful insight into the actual physics of angular momentum transport in accretion disks. As computer power increases and the codes become more sophisticated a steady progress is to be expected. However, at this time there are significant limitations. The numerical accretion flows are relatively thick, the outer boundary is not very far out, the cooling processes are not included, the time integrations can be carried out for a rather modest multiple of the dynamical time scale. Hence, when it comes to modeling disk spectra, conventional steady state, geometrically thin disk models are still commonly used adopting the classical 'no torque' inner boundary condition at $r_{m s}$ (e.g. Blaes, Hubeny, Agol, \& Krolik 2001). The current ADAF or CDAF or variants of Blandford - Znajek models do not exceed a toy model level. This is both, natural and useful, and the quantitative understanding will be improved with time. For the same reason it is useful to have a good quantitative understanding of semi-analytical models of geometrically thin accretion disks. The simple toy models will remain useful even when the future 3-D numerical approach provides full quantitative comparison with the future observations.

At the toy model level one makes a simplifying assumption in order to construct geometrically thin disk, to bypass the issue of thermal (or in general internal) energy balance. Krolik (1999) and Gammie (1999) assumed that the relative disk thickness is constant and small, $H / r=$ const $\ll 1$, even inwards of the marginally stable orbit, in the so called 'plunging region'. We use an alternative assumption: the effective speed of sound is constant and small: $v_{s} / c=$ const $\ll 1$. The two assumptions are roughly equivalent as a small effective speed of sound implies small disk thickness, and vice versa, as long as the flow is in a hydrostatic equilibrium in the 'vertical', i.e. ' $z$ ' direction. This holds even in the supersonic flow, as long as radial velocity is much smaller than rotation velocity, i.e. as long as the accretion time scale is much longer than dynamical time scale.

In our view the essence of a thin disk concept is the assumption that it is OK to integrate disk structure over its thickness, and to consider all important variables to be functions of radius only. In particular, thin disk radiates away energy dissipated locally, and no advection is allowed. In effect a diversity of local physical processes can be ignored, and the conservation laws of mass, angular momentum and energy determine everything. A simplifying ad hoc assumption, be it $H / r=$ const $\ll 1$, or $v_{c} / c=$ const $\ll 1$, is added in order to follow a transition from a subsonic flow at large radii to supersonic infall at small radii.

The argument presented by Paczyński (2000) in favor of applicability of a no torque inner boundary condition was very simple. Geometrically thin, steady state disk accretion 
onto a black hole was sub-sonic at $r>r_{i n}$, and supersonic at $r<r_{i n}$, with the inner disk radius $r_{i n}$ located near the marginally stable orbit, at $r_{m s}$. The specific angular momentum at $r_{i n}$ is $l_{i n}$, and it satisfies the equation

$$
\frac{l_{\text {in }}-l_{0}}{l_{\text {in }}} \approx \alpha \frac{H_{\text {in }}}{r_{\text {in }}} \ll 1, \quad \text { if } \quad \alpha \ll 1 \quad \text { and } \quad \frac{H_{\text {in }}}{r_{\text {in }}} \ll 1
$$

(cf. eq. 4 in Paczyński 2000), where $l_{0} \approx l_{\text {in }} \approx l_{m s}$ is the angular momentum integration constant, $H$ is the disk thickness, and $\alpha$ is the viscosity parameter. Eq. (1) follows from angular momentum conservation in a steady state accretion flow. Current 3-D numerical calculations for thick disks and tori indicate $\alpha \approx 0.1$, with large fluctuations of all physical quantities. The inequality $\left(l_{i n}-l_{0}\right) / l_{\text {in }} \ll 1$ is equivalent to the so called 'no torque' inner boundary condition, or more precisely to a very small torque at the inner disk edge. This conclusion remains valid even for $\alpha \sim 1$, as long as $H_{i n} / r_{i n} \ll 1$, i.e. as long as the disk remains geometrically thin at the critical point. Note that eq. (1) is local, i.e. it does not matter if the parameter $\alpha$ is constant throughout the flow, or does it vary with radius. The two essential assumptions are that the disk is geometrically thin, and that the accretion is steady state.

Please note: we do not use eq. (1) in our numerical model calculation, in which we make no assumption about a relation between $l_{i n}, l_{m s}$ and $l_{0}$. The value of the angular momentum constant is calculated adopting two assumptions: the disk is 'Keplerian' at large radii, and the flow passes smoothly through the effective sonic point. These two conditions select a unique value of $l_{0}$, which turns out to be very close to $l_{m s}$. Also, the effective sonic radius $r_{c}$ is found to be close to the marginally stable orbit, $r_{m s}$.

What can be wrong with eq. (1)? There are several possibilities. Thin disks, with $H_{\text {in }} / r_{\text {in }} \ll 1$ may be physically impossible. We do not know if this is true or not, as we have neither theoretical nor observational proof either way. Steady state accretion may not exist in nature. Current 3-D simulations have indicated that strict steady state flow is not possible, but there is a possibility that when averaged over moderate time scale a quasi steady state may still be a sensible approximation. The eq. (1) may hold as long as the fluctuating disk thickness remains small at all time. This domain is not accessible to numerical calculations so far. Next, there is a global problem pointed out by Abramowicz \& Kato (1989): an accretion flow may pass through a sonic point near $r_{m s}$ but it may not continue all the way into a black hole, as shown schematically in their Fig. 2. In fact, we could not find any reference from 1980s demonstrating that the supersonic infall for $r<r_{m s}$ continues all the way. This was assumed to be obvious and the global flow pattern was not verified numerically.

There is an issue of terminology: what does it mean that a disk is geometrically thin? 
In the eq. (1) the disk thickness $H$ refers to the structure which carries stresses. If the stresses which make the accretion possible are magnetic, as seems to be very likely, then $H$ must refer to the geometrical thickness of the magnetic field structure, not just the gas layer. The notion that the magnetic thickness may be larger than gas thickness dates back at least to Galeyev et al. (1979), and has found some support in recent 2-D and 3-D simulations. Even more importantly, all Blandford - Znajek type models are based on large scale magnetic structures to transfer momentum and energy. The distinction between the magnetic and gas disk thickness was not considered at all in the classical papers about disk accretion, and it was not mentioned by Paczyński (2000). Yet, it is the scale height of the stresses that is important for eq. (1). Therefore, we emphasize that the term: 'geometrically thin disk' refers to the thickness of magnetic structures responsible for the momentum transfer.

Another source of ambiguity comes with the terms 'pressure' and $\alpha$. There are many different ways in which the parameter $\alpha$ has been defined in the literature. In the original work of Shakura \& Sunyaev (1973), $\alpha$ was defined as the ratio of tangential stress to the pressure of the viscous fluid. Balbus \& Hawley (1991), recognized that this stress is in fact dominated by the magnetic terms. Early simulations seemed to indicate that the gas pressure dominates the magnetic pressure, at least outside the marginally stable orbit. Recent 3-D simulations (e.g. Hawley \& Krolik 2002) show that magnetic pressure may dominate the gas pressure inside the marginally stable orbit. Therefore, we define $\alpha$ in terms of total pressure, including magnetic, so it cannot exceed $\sim 1$. It is not essential for our model to have constant value of $\alpha$, this is just the simplest assumption.

Another ambiguity is caused by the 'speed of sound'. It is conventionally defined as the speed at which gas pressure disturbances propagate, or it may also include the effects of radiation pressure. If the magnetic pressure dominates then the Alfvén speed becomes much larger than the speed of sound. Rather than study the complicated effects of different speeds at which different disturbances may propagate we introduce the 'effective speed of sound' $v_{s}=(P / \rho)^{1 / 2}$, where $P$ is the total pressure, which is relevant for the $v_{s}$. With highly tangled magnetic fields this seems to be a reasonable, though perhaps non-orthodox definition. It is the effective speed of sound (the fast magnetosonic speed if magnetic pressure dominates) that defines the transition from a sub-sonic to a supersonic flow. It is beyond the scope of this paper to provide a rigorous justification of our approach, but we think this simplification is sensible. For a disk to remain geometrically thin it is essential that the effective speed of sound is much smaller than the speed of light, but it does not have to be constant. A theory which would allow us to calculate the effective speed of sound from the first principles does not exist. Therefore, we consider the simplest possible disk model, and we assume that throughout the disk the 'effective speed of sound' remains 
constant, i.e. $v_{s}=$ const. This assumption is not essential, it is just the simplest.

We should note that a key ingredient in the above arguments, and in the analysis of the following sections, is the assumption of a hydrostatic equilibrium in the direction perpendicular to the thin disk. It holds where the accretion time scale is longer than dynamical time scale, i.e. where radial velocity is much smaller than rotation velocity. The 'vertical' hydrostatic equilibrium holds even while the radial flow is supersonic, approximately as long as $r>2 r_{g}$. The consequence of 'vertical' hydrostatic equilibrium is a simple relation between the effective speed of sound $v_{s}$, rotation velocity $v_{\text {rot }}$, disk thickness $H$ and the radius $r: v_{s} / v_{r o t} \approx H / r$. It follows that if the disk is assumed to be thin, i.e. $H / r \ll 1$, then the effective speed of sound must be much smaller than the speed of light, i.e. $v_{s} / c \ll 1$. Therefore, the assumption that the disk is thin implies that magnetic energy is assumed to be efficiently dissipated.

Following all these definitions we present a simple thin accretion disk model in order to demonstrate that there is no problem of the type envisioned by Abramowicz \& Kato (1989), i.e. that once the infall becomes supersonic it never 'turns back'. Our simple numerical model reproduces the basic features of classical thin disks, and demonstrates that the transonic solution may remain unique not only for a saddle, but also for a nodal critical point. But first we recall some concepts of the thin disk models of several decades ago. For the derivation of all equations the reader may consult the paper by Abramowicz \& Kato (1989).

The single most important difference between Newtonian accretion and an accretion onto a black hole is the presence of a marginally stable orbit in the latter. Newtonian gravity varies strictly as a power of radius, therefore binding energy and the 'Keplerian' angular momentum vary as power laws of radius, and this makes self-similarity an acceptable simplifying assumption for some accretion flows. This is not the case in general relativity: at small radii 'Keplerian' quantities are no longer power laws of radius, they do not even vary monotonically. The 'Keplerian' angular momentum and the corresponding binding energy reach a minimum at $r_{m s}$, the radius of a marginally stable orbit. This feature is reproduced with a pseudo-Newtonian potential (Paczyński \& Wiita 1980):

$$
\Psi=-\frac{G M}{r-r_{g}}, \quad r_{g}=\frac{2 G M}{c^{2}} .
$$

For a test particle on a 'Keplerian' circular orbit we have a relation

$$
\frac{d \Psi}{d r}=\frac{G M}{\left(r-r_{g}\right)^{2}}=\frac{v_{K}^{2}}{r},
$$

where $v_{K}$ is the 'Keplerian' rotational velocity. It follows that 'Keplerian' angular 
momentum, $l_{K}$, angular velocity, $\Omega_{K}$, and binding energy, $e_{K}$, are given as

$$
\begin{gathered}
l_{K}=v_{K} r=(G M r)^{1 / 2}\left(\frac{r}{r-r_{g}}\right), \quad \Omega_{K}=\frac{v_{K}}{r}=\left(\frac{G M}{r^{3}}\right)^{1 / 2}\left(\frac{r}{r-r_{g}}\right), \\
e_{K}=\Psi+\frac{v_{K}^{2}}{2}=-\frac{G M\left(r-2 r_{g}\right)}{2\left(r-r_{g}\right)^{2}}
\end{gathered}
$$

At very large radii, $r / r_{g} \gg 1$, the eqs. (2-4) asymptotically become Newtonian, but the differences are large at small radii. In particular, a minimum value of 'Keplerian' angular momentum and binding energy is reached at $r=r_{m s}=3 r_{g}$, just as it does in the relativistic Schwarzschild case. The orbits with $r>r_{m s}$ are stable, while those with $r<r_{m s}$ are unstable, with a marginal stability at $r_{m s}$.

Let us consider the simplest disk model with zero pressure. In this case all streamlines are identical with particle trajectories, i.e. they are nested 'Keplerian' orbits. This structure can be extended to small radii, but all orbits inwards of $r_{m s}$ are unstable: a small perturbation makes a particle spiral into a black hole. Therefore, a zero pressure disk is truncated at $r_{m s}$, it cannot extend inwards of $r_{m s}$. Such a disk has zero geometrical thickness, and it is static, i.e. there is no accretion.

To make disk accretion possible it is necessary to introduce some means of angular momentum exchange between nearby streamlines. In general, this will require a non-zero total pressure $P$, a non-zero effective speed of sound $v_{s} \approx(P / \rho)^{1 / 2}$, and a finite disk thickness $H$. For simplicity we assume the effective speed of sound to be constant:

$$
\frac{H}{r} \approx \frac{v_{s}}{v_{K}} \ll 1, \quad v_{s}=\text { const }, \quad \frac{v_{s}^{2}}{c^{2}} \approx \frac{P}{\rho c^{2}} \ll 1, \quad \Sigma=\int \rho d z,
$$

We remind the reader that pressure $P$ is the total pressure:

$$
P \equiv P_{\text {tot }}=P_{\text {gas }}+P_{\text {rad }}+P_{\text {mag }}+P_{\text {turb }} .
$$

The effective speed of sound $v_{s}$ becomes close to Alfvén speed when the magnetic pressure dominates.

We assume that the stresses responsible for angular momentum transport are proportional to the total pressure and the shear rate. The torque exerted by these stresses across radius $r$ is

$$
g=-\alpha 2 \pi r^{2} \Omega_{K}^{-1}\left(\frac{d \Omega}{d \ln r}\right) \int P d z=-\alpha 2 \pi r^{2}\left(\frac{d \ln \Omega}{d \ln r}\right)\left(\frac{\Omega}{\Omega_{K}}\right) v_{s}^{2} \Sigma .
$$

The dimensionless factor $\alpha$ is assumed to be less than unity, and for simplicity we take it to be constant. Again, just as it was the case with the 'effective speed of sound', the 
assumption that $\alpha$ is constant is not essential, it is just the simplest. Note, that $\Omega$ varies monotonically with radius, i.e. the inner disk rotates faster, and angular momentum is transported outwards.

The $\alpha P$ term, no matter how small, redistributes angular momentum within the disk, and accretion becomes possible. In a steady state each disk element gradually loses its angular momentum, and slowly spirals inwards. For $r>r_{m s}$ the process can be envisioned as a motion along ever smaller nearly 'Keplerian' orbits, with gradually decreasing angular momentum. This brings matter close to $r_{m s}$. Following any additional loss of angular momentum the gas cannot find any 'Keplerian' orbit and must plunge into the black hole along a spiral, approximately conserving angular momentum.

Let us assume a steady state, with the mass accretion rate $\dot{M}=$ const $<0$, and the radial flow velocity $v_{r}<0$ assumed to be a function of radius only. The conservation laws of mass and angular momentum are

$$
\dot{M}=2 \pi r \Sigma v_{r}, \quad g=(-\dot{M})\left(l-l_{0}\right),
$$

where $l_{0}$ is the integration constant (cf. Abramowicz \& Kato 1989). Combining eqs. (6) and $(7)$ we obtain

$$
\left(-v_{r}\right)\left(l-l_{0}\right)=\alpha r v_{s}^{2}\left(-\frac{d \ln \Omega}{d \ln r}\right)\left(\frac{\Omega}{\Omega_{K}}\right) .
$$

It is reasonable to expect that $l_{0} \approx l_{m s}$ (to be verified later), and the last equation may be used to estimate the radial flow velocity in the nearly 'Keplerian' thin disk for $r>r_{m s}$ and $\left|v_{r}\right| / v_{s} \ll 1$.

Note, that in eq. (8) only two quantities vary a lot within the flow: $v_{r}$ and $\left(l-l_{0}\right)$, while the others are either constant or vary slowly. In particular, according to the classical theory of thin accretion disks, near the $r_{m s}$ and inwards of $r_{m s}$ angular momentum is almost conserved. This implies that

$$
\left(-\frac{d \ln \Omega}{d \ln r}\right) \approx 2, \quad \text { therefore } \quad\left(-v_{r}\right)\left(l-l_{0}\right) \approx 2 \alpha r v_{s}^{2}\left(\frac{\Omega}{\Omega_{K}}\right) .
$$

The numerical solutions of the following sections will verify this assumption.

We expect that when matter reaches $r \approx r_{m s}$ any additional loss of angular momentum puts it on a spiral infall toward the black hole, during which energy and angular momentum are approximately conserved, i.e.

$$
e=-\frac{G M}{r-r_{g}}+\frac{v_{r}^{2}}{2}+\frac{v_{r o t}^{2}}{2} \approx e_{m s}=-\frac{c^{2}}{16}, \quad l=v_{r o t} r \approx l_{m s}=3 \sqrt{1.5} \frac{G M}{c}
$$


where $v_{r o t}$ is the rotational velocity component of the infall. This is verified with the numerical integrations in the following sections.

To calculate a transition from the sub-sonic radial flow to the supersonic flow we need the equation of motion (cf. Abramowicz \& Kato 1989)

$$
v_{r} \frac{d v_{r}}{d r}-\left(\Omega^{2}-\Omega_{K}^{2}\right) r+\frac{v_{s}^{2}}{\Sigma} \frac{d \Sigma}{d r}+v_{s}^{2} \frac{d \ln \Omega_{K}}{d r}=0
$$

Differentiating the mass conservation law (eq. 7) we obtain

$$
\frac{v_{s}^{2}}{\Sigma} \frac{d \Sigma}{d r}=-\frac{v_{s}^{2}}{r}-v_{s}^{2} \frac{d \ln v_{r}}{d r}
$$

and combining eqs. (11) and (12) we find

$$
\left(v_{r}^{2}-v_{s}^{2}\right) \frac{d \ln v_{r}}{d \ln r}-\left(\Omega^{2}-\Omega_{K}^{2}\right) r^{2}+v_{s}^{2}\left(\frac{d \ln \Omega_{K}}{d \ln r}-1\right)=0 .
$$

We introduce dimensionless constants and variables defined as

$$
\begin{array}{clc}
a \equiv \frac{v_{s}}{c} \ll 1, & b \equiv \frac{l_{0}}{l_{m s}} \approx 1, & 0<\alpha<1, \\
\omega \equiv \frac{\Omega}{\Omega_{K}}, & v \equiv-\frac{v_{r}}{c}>0, & x \equiv \frac{r}{r_{g}} .
\end{array}
$$

The equation of motion (13) and conservation of angular momentum (9) may be written in dimensionless form as

$$
\begin{gathered}
\frac{d \ln v}{d \ln x}=\frac{1}{\left(v^{2}-a^{2}\right)}\left[\left(\omega^{2}-1\right) \frac{x}{2(x-1)^{2}}+2.5 a^{2} \frac{x-0.6}{x-1}\right], \\
\omega=\frac{1.5 \sqrt{3} b(x-1) v}{x\left[x^{1 / 2} v-2 \sqrt{2} \alpha a^{2}(x-1)\right]},
\end{gathered}
$$

\section{Critical Points, Physical Solutions and their Topology}

We seek a physical solution which has the properties

$$
\begin{aligned}
v / a \ll 1, \quad \omega \approx 1, \quad \text { for } \quad x \gg 3, \\
v=a, \quad \text { for } \quad x=x_{c},
\end{aligned}
$$




$$
v / a \gg 1, \quad \text { for } \quad x \ll x_{c} .
$$

i.e. the flow is sub-sonic and the disk is 'Keplerian' at large radii, the flow passes through a critical point, and it becomes supersonic at small radii.

To better understand the behavior of the physical solutions of eq. (14), and to be able to integrate it numerically near the critical point, we decompose it into two equations

$$
\begin{gathered}
\frac{d \ln v}{d t}=\left(\omega^{2}-1\right) \frac{x}{2(x-1)^{2}}+2.5 a^{2} \frac{x-0.6}{x-1} \\
\frac{d \ln x}{d t}=v^{2}-a^{2}
\end{gathered}
$$

which, together with eq. (15), form a two-dimensional autonomous dynamical system. The variable $t$, is a dummy variable and should not be confused with physical time.

As mentioned earlier, the physical solution must pass a critical point to reach the supersonic regime, otherwise the solution would not be single-valued as the right hand side of eq. (14) diverges. Now, the critical points of eq. (14) are the fixed points of eqs. (17) and an understanding of the nature of these fixed points is necessary for constructing a physical solution.

At any fixed point, the right hand sides of eqs. (17) vanish. Eq. (17b) implies that fixed points are always at $v=a$, as required by eq. (16b). The right hand side of eq. (17a) at the critical point, together with eq. (15) (with $v=a$ ) lead to a complicated algebraic equation for $x$ at a given $b$, or vice versa. The analysis is significantly simplified for small values of $a$ and the corresponding relation between $b$ and $x_{c}$ is shown in Fig. 1. We see that, in general, there are either no fixed points or two fixed points in the system, while at the critical value of $b=1+O(\alpha a)$, there is only a single degenerate fixed point at $x \approx 3$.

The nature of the fixed points can be investigated analytically by linearizing eqs. (17) around each of them. It turns out that the left fixed point (smaller value of $x$ ) is always saddle-like, i.e. the two eigenvalues have opposite signs. The right fixed point is of the spiral type (two complex eigenvalues) when the two fixed points are far apart, but it switches to the nodal type (two eigenvalues of the same sign) as the two fixed points get closer. This behavior is shown in Figs. 2-5 which is the typical behavior of a saddle-node bifurcation (Guckenheimer \& Holmes 1983).

The integrations presented in Figs. 2-5 were calculated numerically, except near the critical points, where an analytic expansion was used. All four figures have the same disk parameters: $a=v_{s} / c=0.01$ and $\alpha=0.15$. They differ in the adopted value of the angular momentum constant $b$. The family of solid lines shown in each figure represents possible 
solutions of the eq. (14). The solutions of special interest for us are those which satisfy boundary conditions $(16 \mathrm{a}, \mathrm{b}, \mathrm{c})$. As it turns out none of these figures has a desired solution, but the figures present the changes in the structure of the critical points.

Fig. 2 presents a saddle critical point at $x_{c}=2.7$, and a spiral point at $x_{c}=3.4$. The Phase Portraits for $b>0.9997$ (including $b>1$ ) is qualitatively similar to Fig. 2. The Phase Portrait changes when the angular momentum constant $b$ is reduced, as shown in Fig. 3, where the two critical points are saddle at $x_{c}=2.94$, and nodal at $x_{c}=3.1$. When $b$ is reduced down to $b=0.9947$ the two critical points merge, as shown in Fig. 4 . This corresponds to the point marked $\left(x_{c}, 1\right)$ in Fig. 1 . When $b$ is reduced even more then there is no critical point, as demonstrated with Fig. 5, and as was anticipated (cf. Fig. 1).

A prominent feature of Figs. 2-5 is the 'Keplerian' solution. In the sub-sonic regime, $(v \ll a)$, the the right hand side of eq. (14) is very large for small values of $a$ unless $\omega \simeq 1$ (almost 'Keplerian' angular velocity). This implies that the only slowly varying solution of eq. (14) is an almost 'Keplerian' one. The radial velocity for a 'Keplerian' solution is obtained by setting $\omega=1$ in eq. (15),

$$
v_{0}=\frac{2 \sqrt{2} \alpha a^{2} x}{x^{3 / 2} /(x-1)-1.5 \sqrt{3} b}+O\left(a^{4}\right) .
$$

Figs. 2-5 show that all the other solutions tend to merge with the 'Keplerian' solution as $x$ decreases. This implies that the behavior of the physical solution close to $r_{m s}$ is almost independent of the boundary condition at large radii, since all different solutions merge as they move inwards. Therefore, it is easy to satisfy the boundary condition given with eq. (16a). However, none of the solutions shown in Figs. 2-5 satisfies all three conditions given by eqs. $(16 a, b, c)$.

It is clear that a physical solution may pass through a saddle or a nodal fixed point, but not a spiral. Furthermore, we assume that a physical solution is analytic. This singles out only two ways of passing a nodal fixed point (slow or fast modes), because a linear superposition of fast and slow modes is non-analytic (when $v$ is expressed as a function of $x$ ). In the case of a saddle fixed point, only two solutions (in the direction of two eigenvectors) can pass the fixed point. A conclusion that can be drawn from this consideration is that, in general, the 'Keplerian' solution does not analytically pass the fixed points and only for discrete values of the angular momentum constant $b$ a physical solution is possible.

Now, we are ready to analyze different topologies that can occur in the parameter space of our model. For a given value of $a$ the nature of critical points can be calculated analytically for any pair of values of $\alpha$ and $x_{c}$, or equivalently $\alpha$ and $b$. Fig. 6 shows the possibilities for $a=v_{s} / c=0.01$. The horizontal axis, $x$, is the position of a fixed point and 
the vertical axis is the parameter $\alpha$ that appears in the viscosity model (eq. 5). The fixed points are of the saddle-type, on the left side of the dashed region, of the nodal type inside the dashed region, and of the spiral type on the right side of the dashed region. The solid curve aBCd shows the position of physical solutions that allow passage of the 'Keplerian' curve through the critical point. The critical points present in Figs. 2-4 are indicated in Fig. 6 with diamonds numbered 2, 3, 4, 3, and 2, correspondingly, with $\alpha=0.15$ for all of them. The diamonds with numbers 7,9 , and 11 , correspond to critical points presented in Figs. 7, 9, 11; they are all located on the solid line aBCd.

We note that for $\alpha$ smaller than some critical value $\alpha_{S N}$, the physical critical point is of the saddle type (segment $\mathrm{aB}$ ), while for larger values of $\alpha$ it is of the nodal type (segment $\mathrm{Bd}$ ). The value of $\alpha_{S N}$ depends on $a$. For example for $a=0.01$ we have $\alpha_{S N}=0.08$ (cf. Fig. 6).

A critical point of the saddle type, shown in Figs. 7 and 8, is the type that is usually encountered in astrophysics. The best known examples are the solar wind (Parker 1958) and Roche lobe overflow in binary stars. As argued above, when the parameters $a$ and $\alpha$ are fixed then physical solution exists only for a unique value of $b$ and the corresponding value of $x_{c}$.

In the case of a nodal critical point, the passage is possible through fast or slow ${ }^{1}$ directions. However, a generic solution, which is a combination of fast and slow modes, passes the critical point in the slow direction since the slow solution dominates the fast one close to the critical point. In this sense, a physical solution that passes a nodal point in the fast direction, is unique, similar to the saddle critical point. This kind of passage is seen in the BC section of the aBCd curve in Fig. 6 and and example of the phase portrait is shown in Figs. 9 and 10. Note that, again similar to the saddle point solutions, there is a smooth transition from the sub-sonic to the supersonic regime.

Finally, the physical solution may pass the critical point in the slow direction. However, as argued above, this does not fix the value of $b$ since it is the generic behavior of the solutions, and only by requiring the analyticity of the physical solution one may determine $b$ uniquely. The points on the Cd segment in Fig. 6 are of this type, and an example of the phase portraits is plotted in Figs. 11 and 12.

The transition from the former type of nodal passage to the latter occurs at $\alpha_{N N}$. For example, for $a=0.01, \alpha_{N N} \simeq 0.14$ (point $\mathrm{C}$ in Fig. 6). The curve aBCd, at the transition point $\mathrm{C}$, is tangent to the boundary of the nodal and saddle regions, where the fast and

\footnotetext{
${ }^{1}$ The fast/slow direction refers to the direction of the eigenvector with the larger/smaller eigenvalue.
} 
slow directions merge.

The main difference between the last type of passage and the previous two types is that there is a sharp change in slope, right after passing the critical point. The reason is that the 'Keplerian' curve, which is connected to the slow direction, turns from stable (as $x$ decreases) in the sub-sonic regime to unstable in the supersonic regime, and so the physical solution departs from the 'Keplerian' curve after the passage. Since the slopes are significantly larger far from the 'Keplerian' curve, there is a sharp change in slope.

While we have described some properties of solutions with a nodal critical point, a general discussion of these matters is beyond the scope of this paper. The central issue for us is the inner boundary condition for a steady state geometrically thin accretion disk. For a given value of the effective speed of sound $v_{s}=a c$, and for $\alpha \leq 0.14$ we found a unique numerical solution, in agreement with the finding of Artemova et al. (2001). We verified the uniqueness of numerical solution in two ways. First, we began with a guess of the location of the critical point $x_{c}$, and we searched for the value which provided a solution close to 'Keplerian' at large radii. This provided a unique value of the angular momentum constant $b$ for the given $a$ and $\alpha$. We also started numerical integrations of the eqs. (17 and 15) at large radii, where we adopted a 'Keplerian' model. We searched for the value of the angular momentum constant $b$ for which the solution would pass through the critical point. There was a unique value of $b$ and $x_{c}$ found for the given $a$ and $\alpha$ and it was identical to that found with the first method. There was no difference in the numerical procedure for solutions which had a saddle or a nodal critical point.

We found that for $a=0.01$ and $0.01 \leq \alpha \leq 0.14$ there are unique solutions for the sub-sonic, steady state flow with the effective speed of sound assumed to be constant. The critical point location varied monotonically in the range $2.8286 \leq x_{c} \leq 3.1058$ and the angular momentum constant varied monotonically in the range $1.00068 \leq b \leq 0.99528$, i.e. we had $x_{c} \approx x_{m s}=3.0$ and $b \approx 1.0$. These results are not in any way affected by the supersonic flow at $x<x_{c}$, i.e. no matter how complicated is the flow for $x<x_{c}$ it has no influence on the flow for $x \geq x_{c}$. However, there might be a problem of the type presented by Abramowicz \& Kato (1989) in their Fig. 2: the flow passing through the critical point may formally reverse at some radius $x<x_{c}$, i.e. it may be globally impossible. This is not the case if the effective speed of sound is assumed to be constant also in the supersonic flow: our solutions continue smoothly all the way to the black hole, and the angular momentum remains almost constant within the supersonic flow, as expected.

We were not able to find steady-state solutions for $\alpha>0.14$. Perhaps physical solutions cannot pass a nodal point in the slow direction, and the accretion is non steady for $\alpha>0.14$. We shall explore the consequences of variable effective speed of sound in section 5.2. 


\section{Some Analytic Results}

In order to have an analytic understanding of the behavior of the solutions and the dependence on the parameters $\alpha$ and $a$ we attempt to replace eq. (14) by a simplified version of it, which retains the main topological features of the phase portrait, i.e. critical points and the 'Keplerian' curve, and also the dependence on $\alpha$ and $a$ for small $a$ 's. First let us define $\nu=v / a$ and $\nu_{0}=v_{0} / a$, where $v_{0}$, defined in eq.(18), is the 'Keplerian' radial velocity. Next we make the following assumptions

$$
x \simeq 3, \nu_{0} \simeq 1, \omega \simeq 1
$$

which all follow from the assumption of $a \ll 1$ and restricting the study to the vicinity of the 'Keplerian' curve (that includes the critical points). After these substitutions, being careful not to drop terms that are crucial for the main topological features, we end up with

$$
\frac{d \nu}{d x} \simeq-\frac{\alpha\left(\nu-\nu_{0}\right)}{\sqrt{6} a \nu_{0}(\nu-1)}
$$

We see that the 'Keplerian' curve, for which $d \nu / d x$ vanishes, and the critical points, where $\nu=\nu_{0}=1$, are preserved.

Next, we try to find a workable approximation for $\nu_{0}(x)$. To do so, we notice that the interesting transitions in the nature of the physical solution (Fig. 6) happens where the critical points are close to the maximum of $\nu_{0}(x)$, which is also close to 1 . With these approximations, we can use eq. (18) to get

$$
\frac{d \ln \nu_{0}}{d x} \simeq \frac{d \nu_{0}}{d x} \simeq 1 / 3-2 B(x-3)
$$

where

$$
B^{-1}=32 \sqrt{6} \alpha a
$$

which can be integrated to give

$$
\nu_{0}(x) \simeq 1+B\left[\left(x_{c}-x_{b}\right)^{2}-\left(x-x_{b}\right)^{2}\right], x_{b}=3+B^{-1} / 6 .
$$

$x_{c}$ is the position of the critical point, which is related to $b$ by setting $\omega=1$ in eq.(15), and $x_{b}$ is the Saddle-Node bifurcation point, the boundary of the Saddle region and the Nodal region in Fig. 6.

Now, let us define the new variables $\xi, \Delta$ and $\mu$

$$
\xi \equiv B^{1 / 2}\left(x-x_{b}\right), \mu \equiv B^{1 / 2} \sqrt{6} a / \alpha=\left[\frac{\sqrt{6} a}{32 \alpha^{3}}\right]^{1 / 2}, \Delta \equiv \nu-\nu_{0} .
$$


In terms of these new variables, eq.(20) becomes

$$
\frac{d \Delta}{d \xi}=-\frac{\mu^{-1} \Delta}{\left(1+\xi_{c}^{2}-\xi^{2}\right)\left(\Delta+\xi_{c}^{2}-\xi^{2}\right)}+2 \xi .
$$

Now, it is easy to find the eigenmodes $M$ of the critical point, by setting $\Delta$ equal to $M\left(\xi-\xi_{c}\right)$ and requiring that it satisfies eq. (25) to the first order in $\xi-\xi_{c}$. This yields

$$
M=-\frac{1}{2 \mu}\left(1-4 \mu \xi_{c} \pm \sqrt{1-8 \mu \xi_{c}}\right)
$$

which already gives the three saddle, nodal and spiral regions in Fig. 6.

We do not intend to elaborate any further on the behavior of the solutions of eq.(25). This is mainly because the main property of the physical solution which is connecting the asymptotically 'Keplerian' curve to the critical point, is a global property and so its analytical investigation is not straightforward. The only clear conclusion that we can draw from eq. (25) is that the value of $\xi_{c}$ for which the physical solution exists is a function of $\mu$. The transitions in the nature of the critical point, points $\mathrm{B}(\mathrm{SN})$ and $\mathrm{C}(\mathrm{NN})$ in Fig. 6, that were discussed in the last section, occur at specific values of $\mu$ which can be already fixed from the numerical results in Fig. 6 and the definition of $\mu$ in eq. (24)

$$
\mu_{S N} \simeq 1.22, \mu_{N N} \simeq 0.53
$$

Of course the advantage is that now we can apply this result to all small values of $a$ and not only $a=0.01$. This yields

$$
\begin{array}{cc}
\left(\frac{a}{0.01}\right)<\left(\frac{\alpha}{0.14}\right)^{3}, & \text { Nodal Point Through Slow Direction, } \\
\left(\frac{\alpha}{0.14}\right)^{3}<\left(\frac{a}{0.01}\right)<\left(\frac{\alpha}{0.08}\right)^{3}, & \text { Nodal Point Through Fast Direction, } \\
\left(\frac{\alpha}{0.08}\right)^{3}<\left(\frac{a}{0.01}\right), & \text { Saddle Point. }
\end{array}
$$

The numerical values calculated for $a=0.02$ agree very well with these scalings.

\section{Discussion}

After numerical and analytical study of the properties of the solutions of eq. (14) in the last two sections, we are now ready to discuss the physical picture within the framework of our approximations. Of course the question of accuracy of these approximations should be addressed as well, and we intend to do so, at least in part. 
Our numerical solutions fully confirm our guesses that the angular momentum constant $l_{0} \approx l_{m s}$ (i.e. $b \approx 1$ ), and that $d \log \Omega / d \log r \approx-2$ near $r_{m s}$ and for $r<r_{m s}$ (cf. eq. 9 , and the text between eq. 8 and 9 ). Note: we have not assumed that $l \approx l_{m s}$, this was obtained numerically as a consequence of two conditions: the disk had to be nearly 'Keplerian' at large radii, and the transition through the sonic point had to be smooth (cf. eqs. 16).

\subsection{Torque at the Sonic Point}

The starting point of this investigation was a recent controversy about the significance of the torque at the sonic point of a thin accretion disk. The traditional picture of Novikov \& Thorne (1973) argues for a 'zero torque' boundary condition at the marginally stable

orbit $r_{m s}$. Historically, the possibility of a significant torque at the sonic point due to strong magnetic fields was first brought up in Page \& Thorne (1974). This issue was pursued more seriously by Krolik (1999), Gammie(1999), Agol \& Krolik (2000).

Our disk model, with a smooth flow passage through the effective sonic point, has a small but non-zero torque there. The most relevant way to quantify the importance of this effect is to look at the energy generation rate due to differential rotation, which follows from the conservation laws:

$$
\frac{d L_{d}}{d r}=g \times\left(-\frac{d \Omega}{d r}\right)=(-\dot{M})\left(l-l_{0}\right)\left(-\frac{d \Omega}{d r}\right),
$$

(cf. eq. 7). In the classical model of a geometrically thin disk with a no torque at $r_{m s}$ and 'Keplerian' rotation for $r>r_{m s}$, eq. (29) may be integrated to obtain

$$
L_{d, 0}=\int_{r_{m s}}^{\infty} \frac{d L_{d}}{d r} d r=(-\dot{M}) \frac{c^{2}}{16}=(-\dot{M}) c^{2} \epsilon_{0}, \quad \epsilon_{0}=\frac{1}{16}
$$

Eq. (29) may be evaluated numerically for our disk model, and the results are shown in Fig. 13 for $a=0.01$. A thick line corresponds to the classical model and two thin lines correspond to our models with $\alpha=0.01$ and $\alpha=0.1$, respectively. The differences are small but noticeable. The total energy released is reduced by fraction of one percent in the disk with $\alpha=0.01$ because the angular momentum constant is slightly larger than $l_{m s}$, with $b=1.00068$. The energy is increased by $2.5 \%$ for $\alpha=0.1$ model, because the angular momentum constant is slightly reduced, with $b=0.99636$.

Next, we calculated the change of accretion efficiency:

$$
\frac{\Delta \epsilon}{\epsilon_{0}}=\frac{L_{d}}{(-\dot{M}) c^{2} \epsilon_{0}}-1
$$


where $L_{d}$ was calculated with eq. (29) for 12 disk models with all combinations of the two parameters: $a=0.01,0.02,0.04$, and $\alpha=0.01,0.05,0.10,0.14$. The results are presented in Fig. 14 as a function of angular momentum constant: $b=l_{0} / l_{m s}$. The important result is that the efficiency of accretion increases with increasing disk thickness, which is proportional to the parameter $a$, and with increasing parameter $\alpha$. Within the range of parameters presented in Fig. 14 the efficiency is increased by up to $11 \%$ of $\epsilon_{0}$, i.e. from $0.0625 \dot{M} c^{2}$ to $0.0694 \dot{M} c^{2}$.

There are two related effects contributing to a small change in accretion efficiency. The fact that angular momentum constant $l_{0}$ is not exactly equal $l_{m s}$ affects energy generation

at all radii, even in the 'Keplerian' outer disk, as it is apparent in eq. (29). In addition, the disk near $r_{m s}$ is not completely 'dark' as it would be if the torque at $r_{m s}$ were exactly zero.

The disk scale height $H$ at the $r_{m s}$ may be estimated as

$$
\left(\frac{H}{r}\right)_{m s} \approx\left(\frac{a c}{v_{r o t}}\right)_{m s}=\left(\frac{8}{3}\right)^{1 / 2} a \approx 1.63 a,
$$

i.e. $a=0.04$ corresponds to $(H / r)_{m s} \approx 0.065$. As we assume the effective speed of sound to be constant, the disk thickness increases with radius, and we reach $H / r=1$ at $r \approx r_{g} /\left(2 a^{2}\right)$. The 'Keplerian' condition at 'large radii' makes sense only for $a \ll 1$, and this limits the range of parameters for which our disk models make sense.

\subsection{Is Nodal Passage in the Slow Direction Physical?}

Inspection of Figs. 2, 3, 4, 7, 8, 9, and 10 shows that the geometry of transonic flow appears to be similar when the critical point is of a saddle type or a nodal type, provided the solution passes the latter in the fast direction. The transition from the saddle to the nodal (fast) geometry is smooth, with no apparent problem. The sub-sonic flow connects well to a 'Keplerian' disk at large radii, and the supersonic flow approaches a free fall at small radii. This trouble free region corresponds to the aBC segment (thick line) in Fig. 6 , which ends at point $\mathrm{C}$, where it contacts the borderline of the fixed points of the spiral type, and where $\alpha=0.14$. Obviously, no physical solution can pass through a spiral point. Above point $\mathrm{C}$ the analytical solutions pass the nodal critical point in the slow direction, and these solutions do not appear to be physical (cf. Fig. 11), as there is a sharp change in the slope of the solution (see the end of Sec. 4). We do not know if they are unstable, or perhaps no truly steady-state flow is possible for $\alpha>0.14$ (for $a=0.01$ ).

The study of the stability of these points with the assumption of constant $v_{s}$ and $\alpha$ is even less satisfactory than the steady state solutions. Nevertheless, Kato, Honma, and 
Matsumoto (1988) show that, within these assumptions, local instabilities occur when

$$
\alpha_{S S} \Omega\left(r_{c}\right)>\left|\frac{d v}{d r}\right|_{c}
$$

where $\alpha_{S S}$ is the Shakura-Sunyaev value of $\alpha,\left(\alpha_{S S}=2 \omega \alpha\right)$. The dotted portion of the the physical curve in Fig. 7, which covers almost all of the sector Cd, shows the critical points that satisfy this criterion. This indicates that the nodal passage in the slow direction is probably unstable. We should point out that, contrary to the conventional picture (originally suggested by Matsumoto et al. 1984), this is not a generic property of the nodal critical points, but a generic property of the passage in the slow direction of the nodal point, where $|d v / d r|_{c}$ in eq. (33) is small.

We cannot prove that the transition through a nodal-type point in the fast direction is the only physically acceptable solution, i.e. that it is unique, but this seems to be likely upon inspection of Figs. 9, 10, 11, 12, and it also agrees with the finding of Artemova et al. (2001). If correct, this could resolve the ambiguity discovered by Matsumoto et al. (1984). Unfortunately, we cannot offer a simple physical explanation for the transition from saddle-type to nodal-type critical points while the parameter $\alpha$ increases. We stress that the physical nature of nodal points is outside the scope of our paper, which is concentrated on the issue of the inner boundary condition for geometrically thin steady state accretion disks.

\subsection{Discussion of Gammie (1999) paper}

There remains the issue which gave rise to this paper: why did Krolik (1999), Gammie (1999) and Agol \& Krolik (2000) claim that the torque at inner boundary may be large even for thin, steady state disks, which remain thin even in the 'plunging region'? Let us consider the case presented by Gammie (1999), as he provides the fullest model calculation. Gammie assumed that ' $\ldots$ the disk is thin, $c_{s}^{2} / c^{2} \ll 1 \ldots$ ', and ' $\ldots$ that $\alpha \ll 1$ so that magnetic fields make a negligible contribution to the hydrostatic equilibrium of the disk ...'. He also writes: 'This picture leads one to consider a steady, axisymmetric inflow close to the equatorial plane of the Kerr metric.' These assumptions are practically identical to ours.

Some of the assumptions we made in this paper, and some made by Gammie are similar: we all assume that geometrically thin steady state accretion disks exist, and that magnetic fields are confined to the disk. Our model, with a constant and small effective speed of sound, implies that the flow remains geometrically thin also in the supersonic region, for $r<r_{c}$. Gammie also assumed that the flow was geometrically thin everywhere. 
In fact his flow thickness, $H$, was decreasing for $r<r_{m s}$ according to $H / r=$ const $\ll 1$ (cf. his eq. 4).

A disagreement appears in the treatment of the transition from the 'disk' to the 'plunging region'. Gammie adopts one set of assumptions for the flow in the 'disk', for $r>r_{i n}$, and a very different set of assumptions for the flow in the 'plunging region', for $r<r_{i n}$. At $r_{i n}$ the model undergoes a dramatic jump in the adopted physical conditions: it is gas dominated and has negligible magnetic field for $r>r_{i n}$, and it has no gas pressure and it is magnetic field dominated for $r<r_{i n}$. No justification is offered for this jump in physical conditions. The location of $r_{i n}$ is adopted to be close to the marginally stable orbit, $r_{i n} \approx r_{m s}$.

We do not introduce any $r_{i n}$, and we treat the 'disk' and the 'plunging region' with the same equations. We require a smooth transition from sub-sonic flow in the 'disk' to supersonic flow in the 'plunging region' through a critical (sonic) point at some $r_{c}$. We make no assumption about the value of $r_{c}$, the condition of a smooth passage determines that value uniquely. We find that the critical point is located close to the marginally stable orbit, $r_{c} \approx r_{m s}$, but it is in general not at $r_{m s}$. The requirement of a smooth passage through the sonic point imposes a stringent restriction on the solution, it acts like a boundary condition.

Another problem with Gammie's model is the assumption that the flow thickness is proportional to radius, i.e. that the flow becomes geometrically thinner in the "plunging region', while the effective speed of sound rapidly increases with the decreasing radius in his model. This is incompatible with the hydrostatic equilibrium in the direction perpendicular to the equatorial plane. In fact there is no reference to the hydrostatic equilibrium in his paper. That equilibrium should hold as long as radial velocity remains much smaller than rotational velocity, i.e. as long as the accretion time scale remains much longer than the dynamical time scale. As the effective speed of sound increases in Gammie's model, the flow thickness should also increase, not decrease. A quasi-static expansion needed to maintain hydrostatic equilibrium is likely to conserve magnetic flux, i.e. $B_{r} H$ and $B_{\varphi} H$, where $H$ is the disk thickness. As $H$ increases the magnetic field gets weaker, $B \sim 1 / H$, and the magnetic torque at a given radius $r$ is reduced: $\sim B_{r} B_{\varphi} r H \sim r / H$.

For the same reason the Alfvén speed $v_{A}$ is reduced by flow expansion, so as to maintain approximate hydrostatic equilibrium: $v_{A} / v_{r o t} \sim H / r$.

It is beyond the scope of this discussion to speculate how the conclusions of AKG about the torque at $r_{i n}$ would change if the two major inconsistencies in Gammie's analysis were corrected: the jump at $r_{i n}$ was replaced with full continuity of all physical quantities, and the consequences of hydrostatic equilibrium in the 'vertical' direction were incorporated 
in the model. These two problems are serious enough to doubt the conclusions: the torque at $r_{m s}$ may be large and the accretion efficiency may exceed $100 \%$.

No direct comparison is possible between our model and Gammie's (1999) model, as the latter does not specify the value of $H / r$. Amazingly, his results appear to be independent of $H / r$ value, while our results are sensitive to the assumed disk thickness, as given with the $a$ parameter (cf. eq. 32, and Fig. 14).

The consequences of ad hoc assumptions may be very diverse. AGK claim the importance of magnetic fields increases with decreasing radius, and becomes dominant in the 'plunging region'. Using different ad hoc assumptions Li (2002) comes to the opposite conclusion in his simple analytic model.

A large part of Agol \& Krolik (2000) analysis remains correct: there may be a torque applied to the inner edge of the accretion disk provided there is a large scale magnetic field, possibly threading the black hole. This is a modified Blandford-Znajek mechanism (e.g. Li 2000, Wang et al. 2002, and references therein). The gaseous disk may remain geometrically thin while the stresses are transmitted by a large scale magnetic field which has a vertical scale height comparable to radius. However, this picture is conceptually very different from the case considered by us and by Gammie (1999), as we and Gammie assume that the magnetic fields are confined to a geometrically thin flow.

\section{Conclusions}

The condition given with the eq. (1) of this paper is local, and it is approximately valid even if the parameter $\alpha$ varies with radius. It requires the assumption of a steady state to hold. However, we think it is reasonable to expect that it may also hold for a disk with an accretion which is steady in a statistical sense only, as long as the disk thickness does not exceed the value $H \ll r$ throughout the fluctuations cycle. Obviously, it is important to verify this claim with the full 3-D time dependent simulations. However, current numerical models are limited by the available computer power to disks which are much thicker than the one considered in this paper. They are also limited by the absence of cooling processes

which are essential for the formation of thin disks. At this time there is no observational or theoretical reason to exclude the possibility that geometrically thin disks exist. Currently, there is also no way to prove their existence, as the relevant thickness is that of the layer over which the magnetic fields transferring angular momentum extend, and this quantity is not readily observable.

We have found a unique steady state solution for a simple model with a constant 
effective speed of sound $v_{s}=0.01 c$, and a given viscosity parameter $\alpha<0.14$. The solution was found by approaching the critical point from large radii, i.e. from the sub-sonic flow side, where at large radii the disk was assumed to be 'Keplerian'. The same solution was found starting integrations from the critical point and proceeding out and seeking a solution approaching 'Keplerian' at large radii. No assumption was made about the value of the angular momentum constant $l_{0}$, which was determined by our choice of boundary conditions: 'Keplerian' disk at large radii, and a smooth passage through the sonic point.

Our numerical model confirms the validity of our eq. (1): we find that in a thin steady state disk the sonic point $r_{c}$ is located close to the marginally stable orbit $r_{m s}$, and the value of angular momentum constant $l_{0}$ is close to $l_{m s}$.

The supersonic flow has no effect on the critical point, as expected on general grounds. In particular, we demonstrated that, for geometrically thin inflow, with constant effective speed of sound, there is no global problem of the type envisioned by Abramowicz \& Kato (1989) in their Fig. 2. Once the flow passes the critical point it plunges into the black hole along a spiral, roughly conserving angular momentum, as asserted in many papers written in the $1980 \mathrm{~s}$.

In our disk model the torque at the sonic point is small, but not exactly zero, and the total disk luminosity is modified, but only by several per cent. This small torque and a modest change of accretion efficiency makes the largest difference near $r_{m s}$, where the accretion flow is no longer 'dark', as it was under strict 'no torque' inner boundary condition. This effect should be included in calculating spectra of thin disks.

We emphasize that for a disk to be thin its thickness $H$ must include the magnetic structures responsible for angular momentum transfer, not just the gas layer. The effective speed of sound $v_{s}$ is affected by the total pressure, which includes the contribution of magnetic pressure (cf. eq. 5b).

Just as discovered by Matsumoto et al. (1984) we also found that the critical point is of the saddle-type for small values of $\alpha$, and becomes a nodal-type for large $\alpha$, in our case for $\alpha>0.08$. Unfortunately, we cannot provide a simple physical explanation for this transition. However, we presented plausible arguments that the transition through the nodal-type critical point is unique, as there is only one solution which appears to be smooth. This was also demonstrated by Artemova et al. (2001).

We found that our steady state solutions exist only for $\alpha<0.14\left(100 v_{s} / c\right)^{1 / 3}$, and it appears that there are no physically sensible solutions for $\alpha>\alpha_{\text {crit }} \approx 0.14\left(100 v_{s} / c\right)^{1 / 3}$. It is not clear if this is a general result or just an artifact due to simplicity of our model. The issue is not the particular value of $\alpha_{\text {crit }}$, which is certainly model dependent, but the 
very existence of $\alpha_{\text {crit }}$. Unfortunately, we cannot offer a simple physical explanation for this finding.

Krolik (1999), Gammie (1999) and Agol \& Krolik (2000) claimed that their geometrically thin steady state disks had large torques at $r_{m s}$, and that accretion efficiency could be larger than $100 \%$ of $\dot{M} c^{2}$, i.e. a thin accretion flow could extract energy from a spinning black hole. This may be correct if there are large scale magnetic fields, like those proposed for the modified Blandford-Znajek mechanism, also referred to as a magnetic coupling model (e.g. Agol \& Krolik 2000, Li 2000, Wang et al. 2002, and references therein). However, if the magnetic fields are confined to a thin accretion flow, as in the model described by Gammie (1999), the very high accretion efficiency is almost certainly a consequence of several inconsistent assumptions (cf. our Sec. 6.3). It would be interesting to learn how Gammie's results change when his errors are corrected, but this is beyond the scope of this paper.

With our thin disk model we found that there is a small torque at $r_{m s}$, and there is a corresponding modest change of accretion efficiency. It is not possible to compare our results directly with those obtained by Gammie as he does not specify how thick his disk is.

We are very grateful to the anonymous referee who relentlessly pressured us to refine our analysis. It is a great pleasure to acknowledge many useful and critical comments by Dr. J. Goodman.

\section{REFERENCES}

Abramowicz, M. A., Jaroszyński, M. \& Kozlowski, M. 1978, A\&A, 63, 209

Abramowicz, M. A., \& Kato, S. 1989, ApJ, 336, 304

Agol, E., \& Krolik, J. H. 2000, ApJ, 528, 161

Agol, E., Krolik, J., Turner, N. J. \& Stone, J. M. 2001, ApJ, 558, 543

Armitage, P. J. 1998, ApJ, 501, L189

Armitage, P. J. 2001, MNRAS, 330, 895

Armitage, P. J. \& Natarajan, P. 1999, ApJ, 523, L7

Armitage, P. J., Reynolds, C. S. \& Chiang, J. 2001, ApJ, 548, 868

Artemova, I. V. et al. 2001, ApJ, 549, 1050

Balbus, A. S. \& Hawley, J. F. 1991, ApJ, 376, 214

Balbus, A. S. \& Hawley, J. F. 1991, ApJ, 376, 214 
Ball, G. H., Narayan, R. \& Quataert, E. 2001, ApJ, 552, 221

Blaes, O. 2002, astro-ph/0211368

Blaes, O., Hubeny, I., Agol, E., \& Krolik, J. H. 2001, ApJ, 563, 560

Blandford, R. D. \& Znajek, R. L. 1977, MNRAS, 179, 433

Cao, X., \& Xu, Y. D. 2002, astro-ph/0211220

Cunningham, C. T. 1975, ApJ, 202, 788

Cunningham, C. T. 1976, ApJ, 208, 534

Flemming, T. P., Stone, J. M. \& Hawley, J. F. 2000, ApJ, 530, 464

Galeyev, A. A., Rosner, R. \& Vaiana, G. S. 1979, ApJ, 229, 318

Gammie, C. F. 1999, ApJ, 522, L57

Guckenheimer, J., Holmes, P., "Nonlinear Oscillations, Dynamical Systems, and Bifurcation of Vector Fields", 1983, Springer

Hawley, J. F. \& Balbus, A. S. 1991, ApJ, 376, 223

Hawley, J. F. \& Krolik, J. H. 2002, ApJ, 566, 164

Hawley, J. F., Balbus, S. A., \& Stone, J. M. 2001, ApJ, 554, L49

Igumenschchev, I. V., Narayan, R. \& Abramowicz, M. A. 2003, astro-ph/0301402

Krolik, J. H. 1999, ApJ, 515, L73

Krolik, J. H. \& Hawley, J. F. 2002, ApJ, 573, 754

Li, L.-X. 2000a, ApJ, 531, L111

Li, L.-X. 2000b, ApJ, 533, L115

Li, L.-X. 2002, astro-ph/0212503

Lin, D. N. C. \& Papaloizou, J. C. B. 1996, ARA\&A, 34, 205

Lynden-Bell, D. \& Pringle, J. E. 1974, MNRAS, 168, 603

Matsumoto, R. et al. 1984, PASJ, 36, 71

Merloni, A. 2002, astro-ph/0210251

Merloni, A. 2003, astro-ph/0302074

Merloni, A., \& Fabian, A. C. 2003, astro-ph/0303143

Muchotrzeb, B. \& Paczyński, B. 1982, AcA, 32, 1

Novikov, I. D. \& Thorne, K. S. 1973, In 'Black Holes - Les Astres Occlus', Eds: C. De Witt \& B. S. De Witt, New York: Gordon \& Breach 
Paczyński, B. 2000, astro-ph/0004129

Paczyński, B., \& Wiita, P. 1980, A\&A, 88, 23

Page, D. N., \& Thorne, K. S. 1974, ApJ, 191, 1974

Parker, E. N. 1958, ApJ, 128, 664

Pringle, J. E. 1981, ARA\&A, 19, 137

Pringle, J. E. \& Rees, M. J. 1972, A\&A, 21, 1

Reynolds, C. S. \& Armitage, P. J. 2001, ApJ, 561, L81

Reynolds, C. S., Armitage, P. J. \& Chiang, J. 2001, 20th Texas Symposium, AIP Conf. Proc. Vol. 586, p. 668 (Eds.: J. C. Wheeler, \& H. Martel) = astro-ph/0102045

Sano, T. \& Inutsuka, S.-I. 2001, ApJ, 561, L179

Shakura, N. I. \& Sunyayev, R. A. 1973, A\&A, 24, 337

Stoeger, W. R. 1976, A\&A, 53, 267

Stone, S. \& Pringle, J. E. 2001, MNRAS, 322, 461

Stone, J. M., Pringle, J. E. \& Begelman, M. C. 1999, MNRAS, 310, 1002

Wang, D. X., Xiao, K., \& Lei, W. H. 2002, MNRAS, 335, 655

Wilms, J. et al. 2001, MNRAS, 328, L27 


\section{A. 'Quasi-adiabatic' accretion}

Our assumption that the accretion flow remains geometrically thin at all radii: in the sub-sonic part for $r>r_{c r}$, and in the supersonic part for $r<r_{c r}$, is physically equivalent to the assumption that magnetic fields dissipate effectively at all radii. In this Appendix we discuss the consequences of a possibility that there is no field dissipation at radii smaller than some transition radius $r_{t r}$. In such a case the magnetic field pressure and the disk thickness may increase for $r<r_{t r}$. Krolik (1999) pointed out the possibility that the differential rotation within the supersonic flow may increase the effective speed of sound so much that '... we expect the Alfvén speed in the fluid frame to be $\sim$ c ...' (Krolik 1999). Obviously, if the magnetic field dominates the flow then the effective speed of sound is the same as Alfvén speed, and it also becomes relativistic.

The largest energetically possible increase of the effective speed of sound may be obtained by assuming a 'quasi-adiabatic' flow: all energy transfered from differential rotation into the accretion flow is used to increase magnetic energy, no magnetic energy is dissipated, and none is radiated away. This is certainly a limit which is not likely to be reached in realistic flows. Nevertheless, we explored this possibility as a limiting case, and we refer to it as a 'quasi-adiabatic' approximation. Note that the model developed by Gammie (1999) was also 'quasi-adiabatic'.

We repeated the calculations of our models with $a=0.01$. In the first case we adopted $\alpha=0.01$, in the second $\alpha=0.1$, and we obtained: $\left(x_{c}=2.82859, b=1.00068\right)$ and $\left(x_{c}=3.04505, b=0.99636\right)$, for the two cases, respectively. Obviously, these values were identical to the models described in Section 4. We continued these solutions some distance into the supersonic part of the flow, down to a transition radius $x_{t r}$, where we abruptly switched the flow from having constant effective speed of sound $v_{s}=0.01 c$, and constant $\alpha$, to a 'quasi-adiabatic' approximation. All physical quantities varied continuously, but their derivatives experienced a jump.

We assumed that at $x_{t r}$ the ratio of magnetic pressure to total pressure was equal to $\alpha$. For $r<r_{t r}$ the energy of differential rotation was pumped into tangled magnetic fields, hence the magnetic pressure increased. No magnetic energy was dissipated, and the gas pressure was assumed to change adiabatically, i.e. the gas pressure decreased because of decompression. Therefore, the ratio $P_{\text {mag }} / P_{t o t}$ increased down the flow for $x<x_{t r}$, and the parameter $\alpha$ increased correspondingly. We assumed that hydrostatic equilibrium was maintained in the direction perpendicular to the disk equatorial plane, as that equilibrium was established on a fraction of rotational period, while the flow followed many rotations prior to crossing the black hole horizon. The gas density rapidly decreased as the flow accelerated toward the black hole and expanded in vertical direction. The vertical expansion 
of the flow was a consequence of the increase in the effective speed of sound, which became practically equal to the Alfvén speed.

We found that if the transition from $v_{s}=a c=$ const to a 'quasi-adiabatic' flow was made sufficiently far into the supersonic region, with $x_{t r}<2.79$ and $x_{t r}<2.78$ for the two models, respectively, the flow proceeded smoothly all the way to the black hole, and remained similar to the flow in our original model. At the transition radius, $x_{t r} \approx 2.8$, the flow was moderately supersonic, with the effective Mach number of 1.3 and 2.4, for the two cases, respectively. Down the flow, for $x<x_{t r}$, the magnetic pressure increased, and the parameter $\alpha$ also increased to $\alpha \approx 1$. The effective speed of sound gradually increased by a factor $\sim 3$, increasing the flow thickness correspondingly, but the flow remained supersonic.

When the transition was made at a larger value of $x_{t r}$, i.e. at a smaller effective Mach number, the flow pattern was initially 'normal', i.e. the $\alpha$, the effective Mach number and the Alfvén speed all increased gradually with decreasing radius. However, at still smaller radii the flow first became sub-sonic, and later the numerical solution 'reversed' the flow direction, changing the sign of $v_{r}$, i.e. the flow proceeded back toward large radii. Obviously, this is physically impossible, as pointed out by Abramowicz \& Kato (1989). Superficially a global steady state solution could not exist for the transition radius larger than approximately $2.8 r_{g}$. However, it is virtually certain that before the flow would 'reverse', the deceleration would generate a shock, and a transition to a sub-sonic accretion. Perhaps a steady state flow could be maintained, with the second sonic point located down the flow from the shock location, and the matter ultimately plunging into the black hole. However, these complexities are beyond the scope of our paper.

The results of our numerical experiment are easy to understand qualitatively. As the dissipation of magnetic energy is switched off ('quasi-adiabatic' approximation) the magnetic pressure builds up, and the flow expands in vertical direction. Had the transition been made at a large radius, the disk would become geometrically thick. The very assumption that the disk is thin is obviously equivalent to the assumption that the dissipation of magnetic field is efficient.

Gammie (1999) assumed that there was no magnetic dissipation in his model i.e. his model was also 'quasi-adiabatic'. But there were major differences between his and our models. Gammie assumed that $H / r=$ const $\ll 1$ at all $r<r_{i n} \approx r_{m s}$, while our flows thickened appreciably for $r<r_{t r}<r_{m s}$. Gammie assumed that magnetic fields had a very special smooth spiral geometry, while we assumed they were tangled. His solutions for the flow were very different from ours. The main result of our experiment is that in a 'quasi-adiabatic' approximation the flow thickens, which is hardly surprising. We also found that properties of a 'quasi-adiabatic' flow strongly depend on the value of the 
transition radius $r_{t r}=x_{t r} r_{g}$. Gammie did not explore this diversity, effectively adopting $r_{i n}=r_{t r}=r_{m s}$

We were curious to study the consequences of a 'quasi-adiabatic' approximation, and we were intrigued to find that even within this approximation our solutions were very different from those obtained by Gammie. However, this experiment is not relevant to the main topic of our paper, which was focused on the properties of thin accretion disk. 


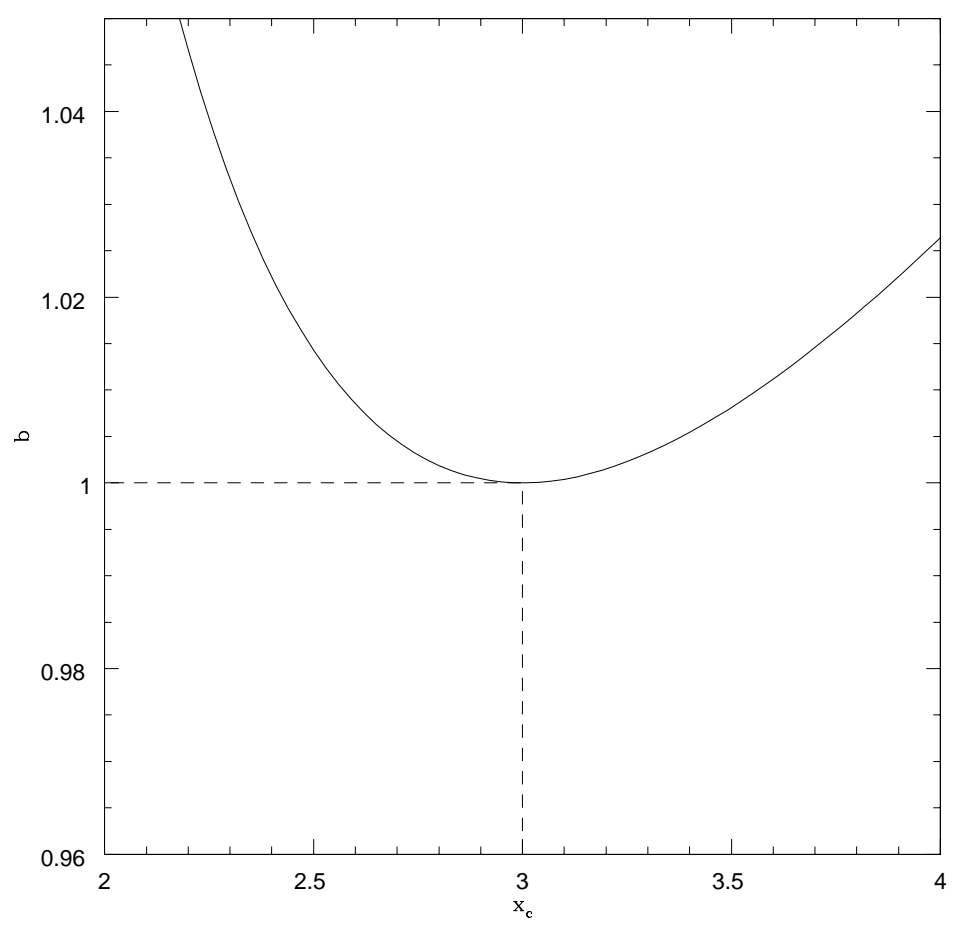

Fig. 1. - The angular momentum constant $b$ is shown as a function of the position of the critical point $x_{c}$ for $a \rightarrow 0$. We see that, for $b>1$, there are two fixed points, at $b=1$, there is one degenerate fixed point at $x=3$ (marginally stable orbit), and for $b<1$ no fixed point exist. 


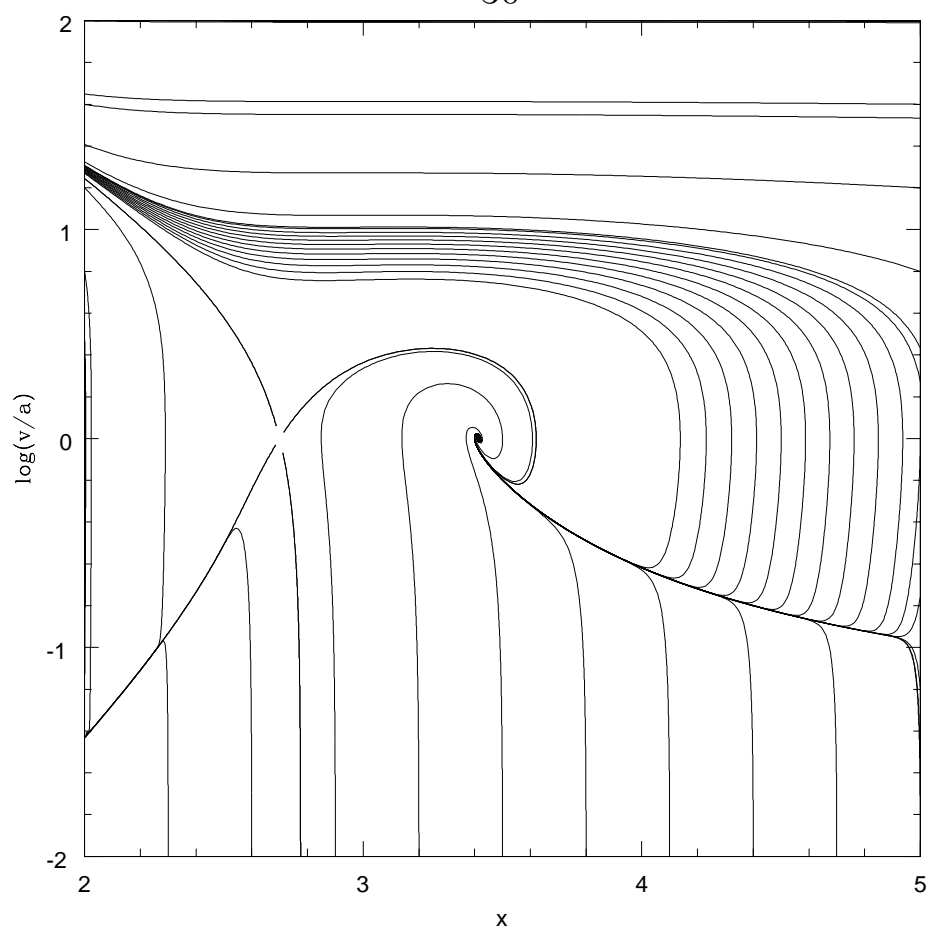

Fig. 2. - The Phase Portrait for $a=0.01, \alpha=0.15$ and $b=0.9997$. There is a saddle point at $x=2.7$ and a spiral point at $x=3.4$.

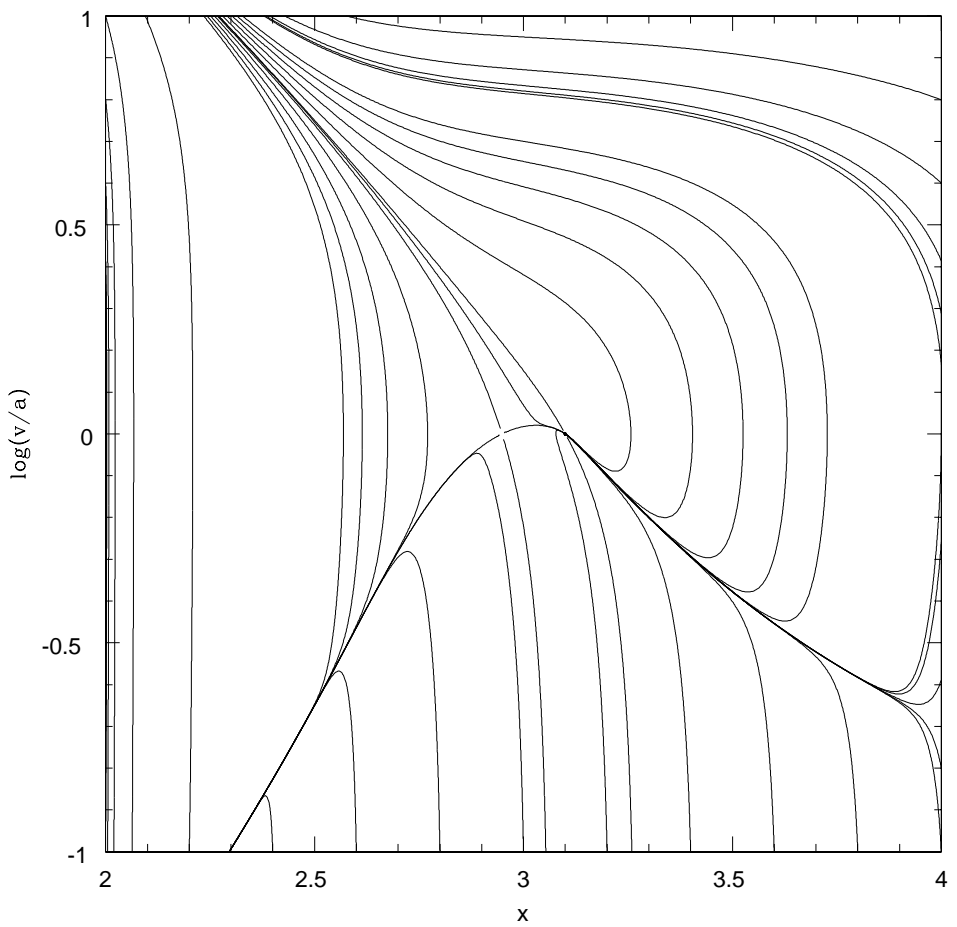

Fig. 3. - The Phase Portrait for $a=0.01, \alpha=0.15$ and $b=0.9949$. There is a saddle point at $x=2.94$ and a nodal point at $x=3.1$. 


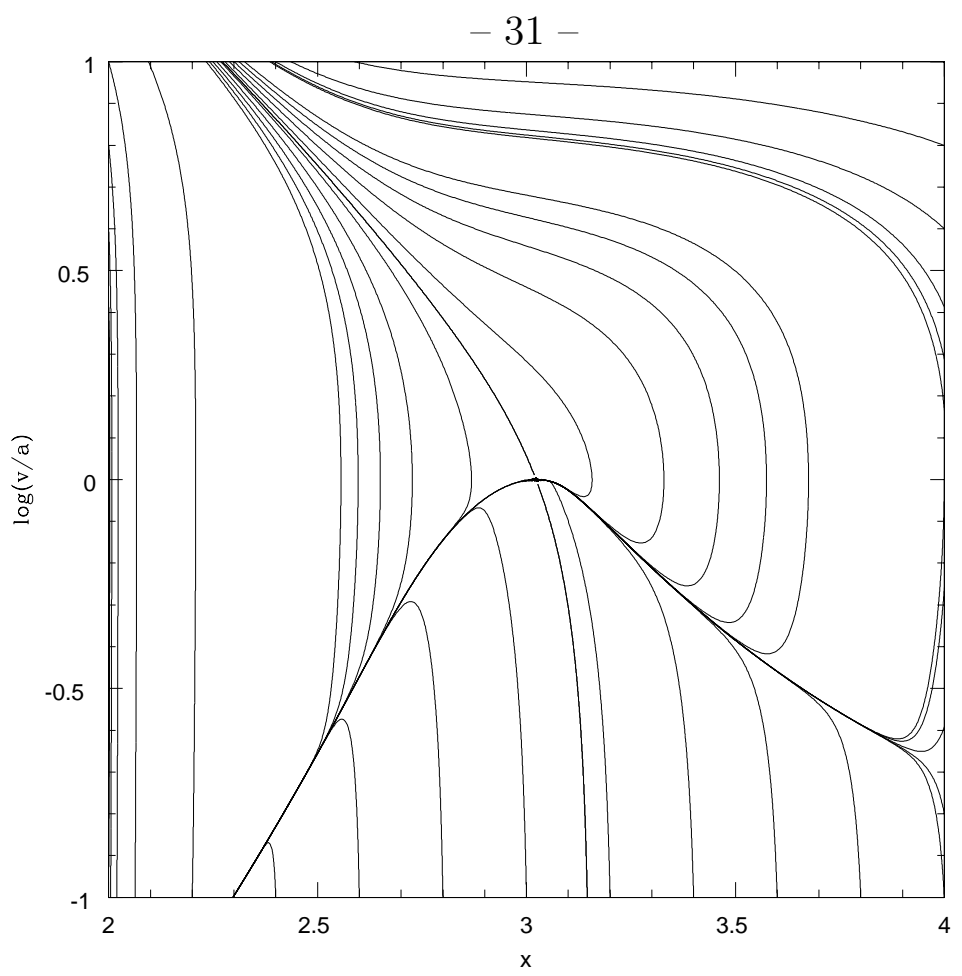

Fig. 4. - The Phase Portrait for $a=0.01, \alpha=0.15$ and $b=0.9947$. There is a degenerate saddle-node point at $x=3.0228$.

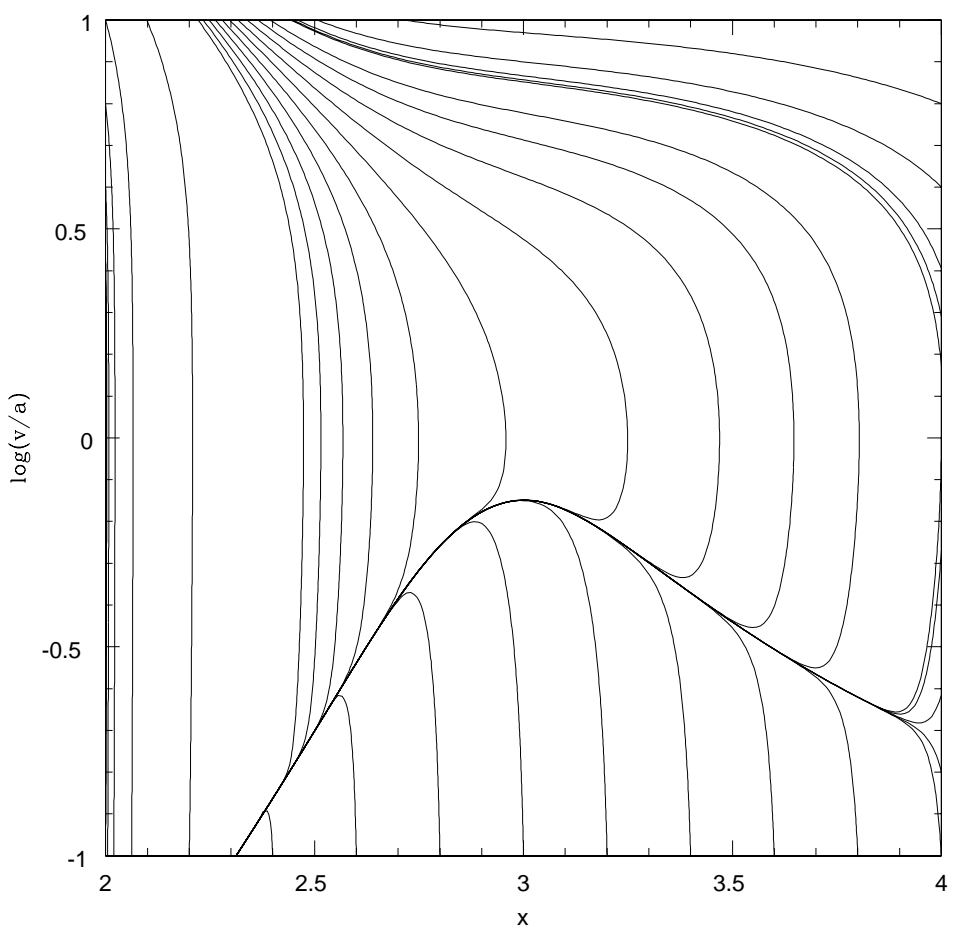

Fig. 5. - The Phase Portrait for $a=0.01, \alpha=0.15$ and $b=0.9927$. There is no fixed point for these parameters. 


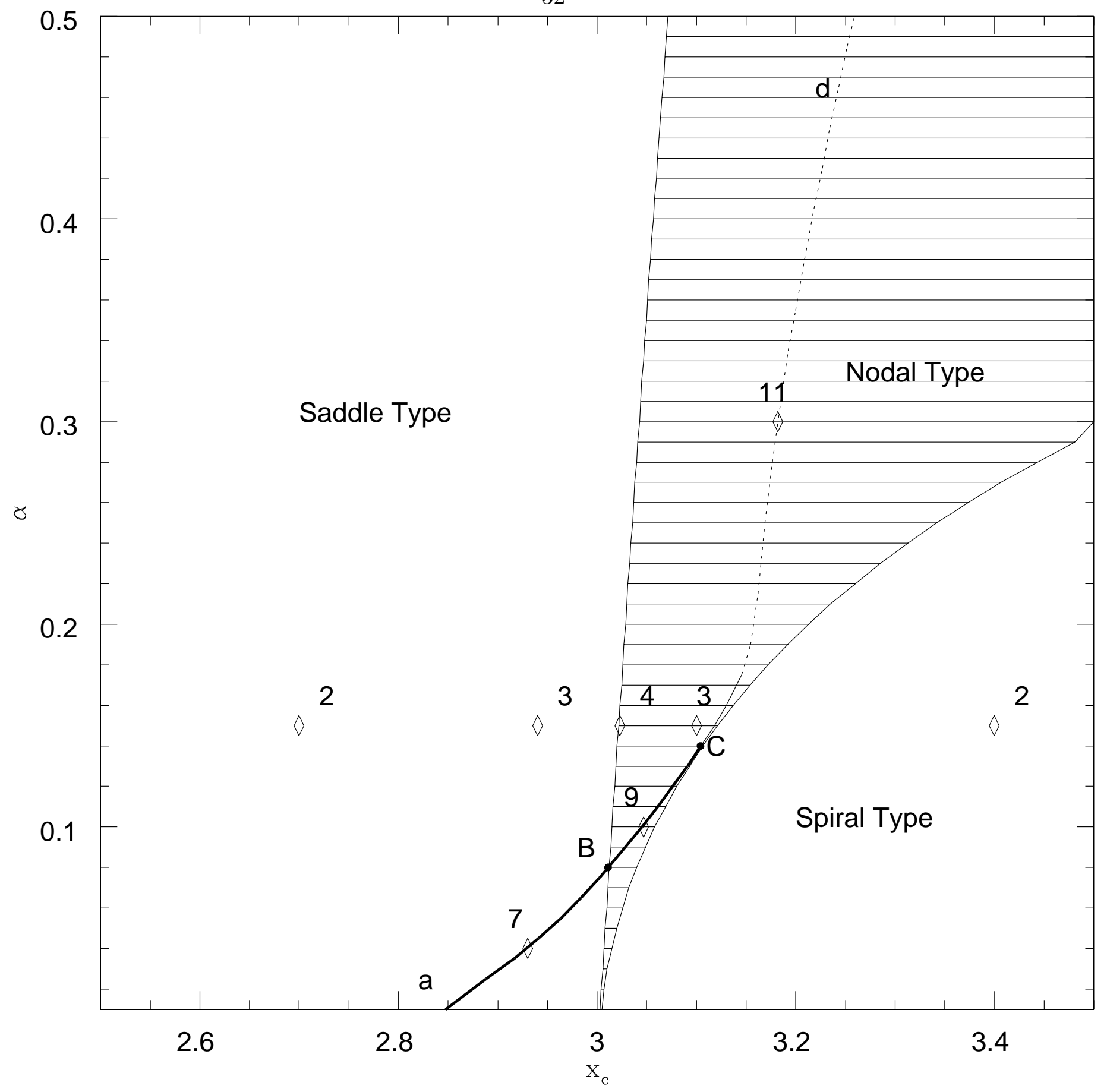

Fig. 6. - The parameter space of the problem for $a=v_{s} / c=0.01 . x_{c}$ is the radius of the critical point in units of $r_{g}$ and $\alpha$ is the viscosity parameter. The curve aBCd shows the position of the fixed points that allow physical solutions (cf. Sec. 4), while the dotted section of the curve are unstable solutions according to eq.(30). Also the parameters of phase portraits in the other figures are represented by numbered diamonds. The number of each diamond is the number of the associated figure in this paper. The Phase Portrait presented in Fig. 5 has no fixed points, and therefore it is not represented in this figure. 


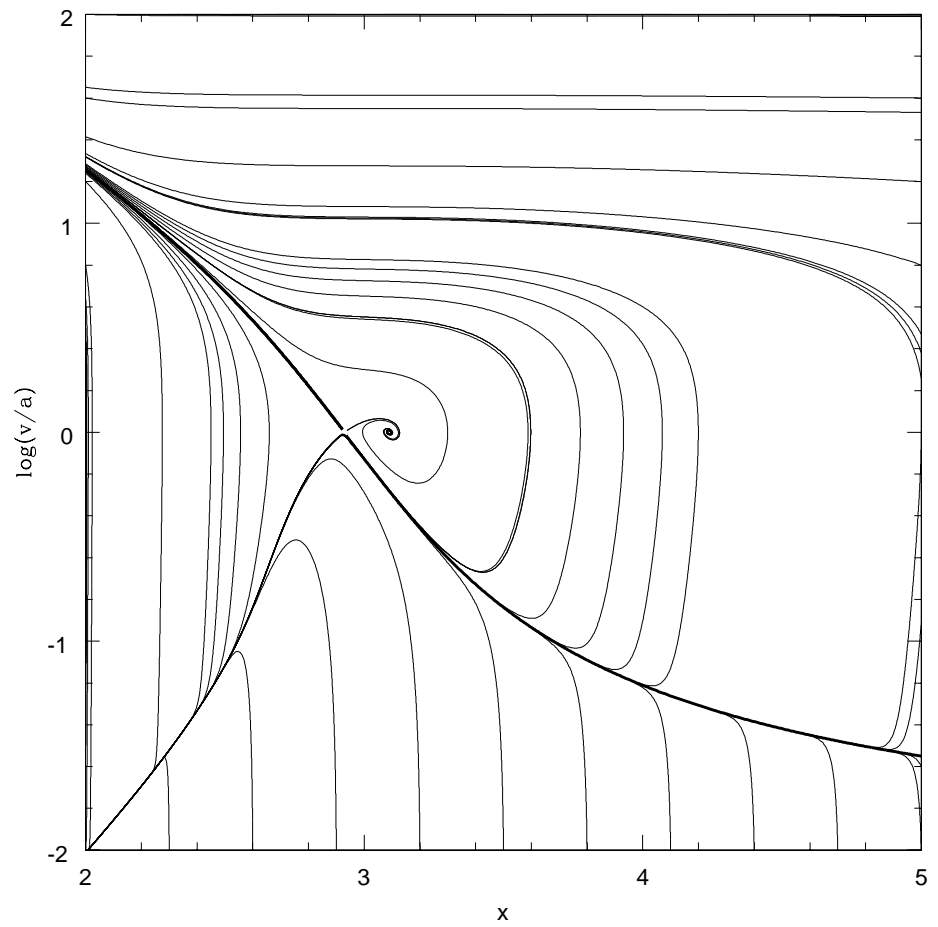

Fig. 7.- The Phase Portrait for $a=0.01, \alpha=0.04$ and $x_{c} \simeq 2.930$. The physical solution (thick curve) passes a critical point of the saddle type.

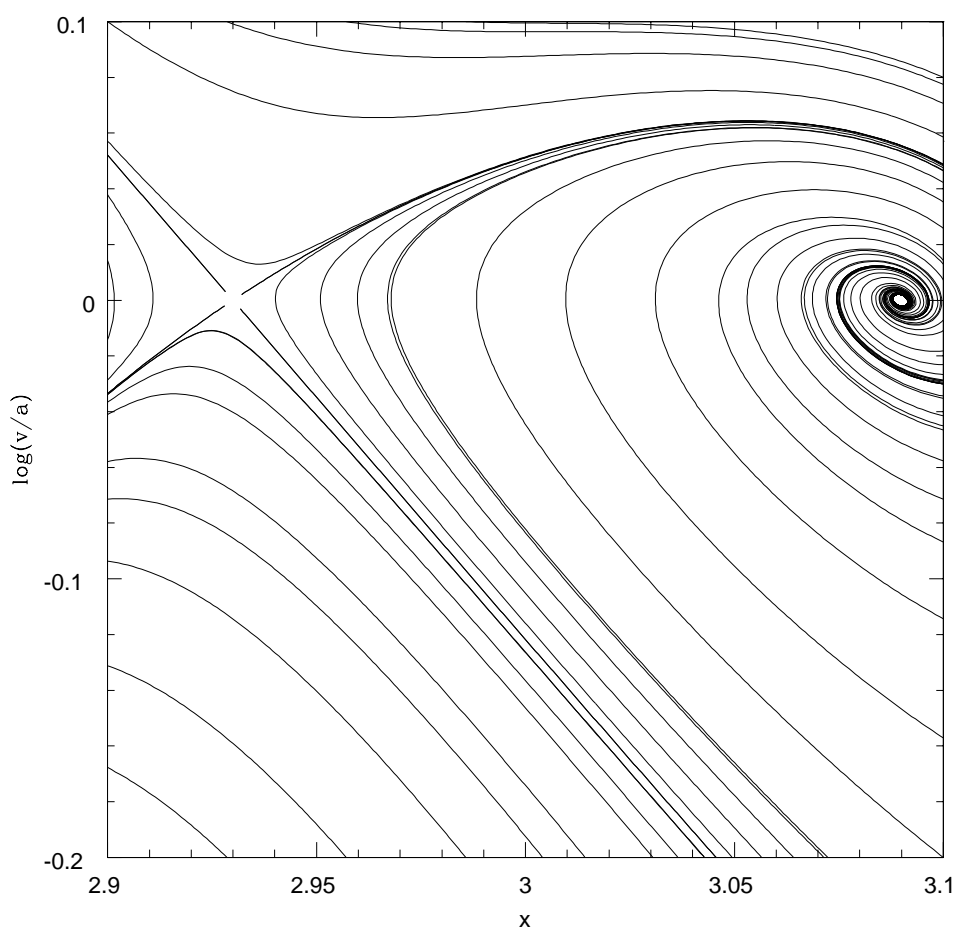

Fig. 8. - The vicinity of the critical points in the phase portrait of Fig. 7. 


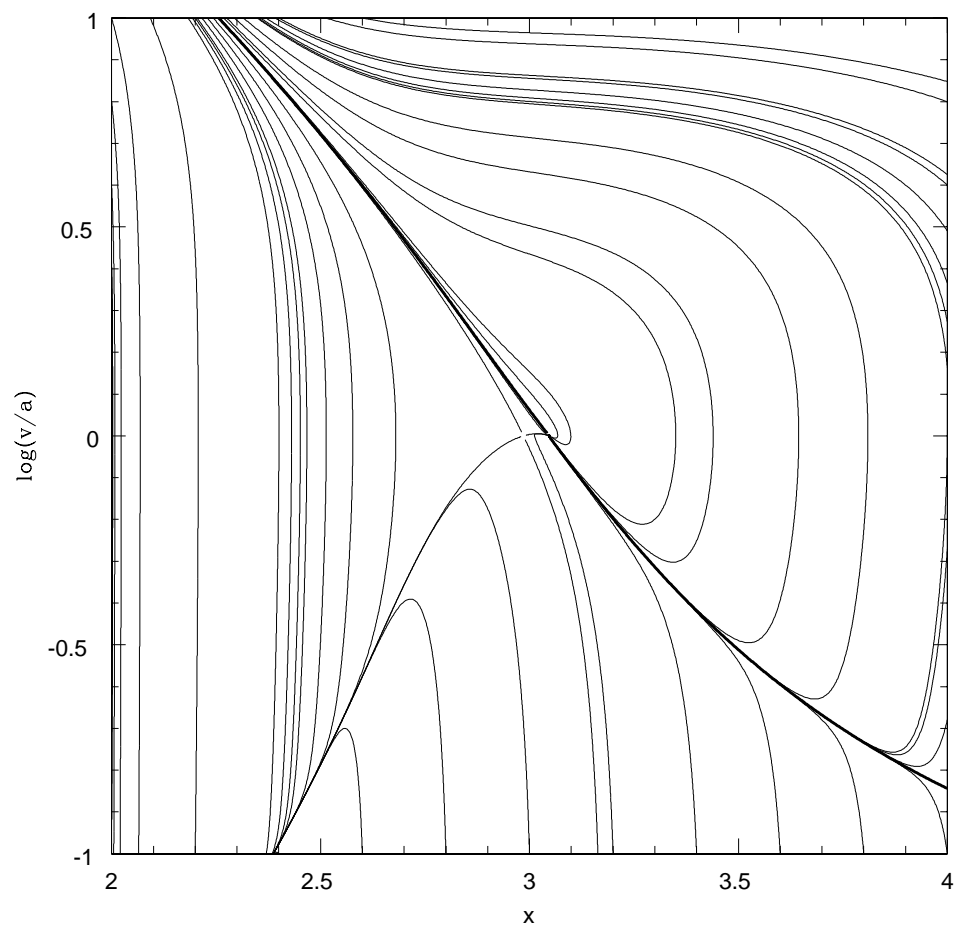

Fig. 9.- The Phase Portrait for $a=0.01, \alpha=0.1$ and $x_{c} \simeq 3.047$. The physical solution (thick curve) passes a critical point of the nodal type, in the fast direction.

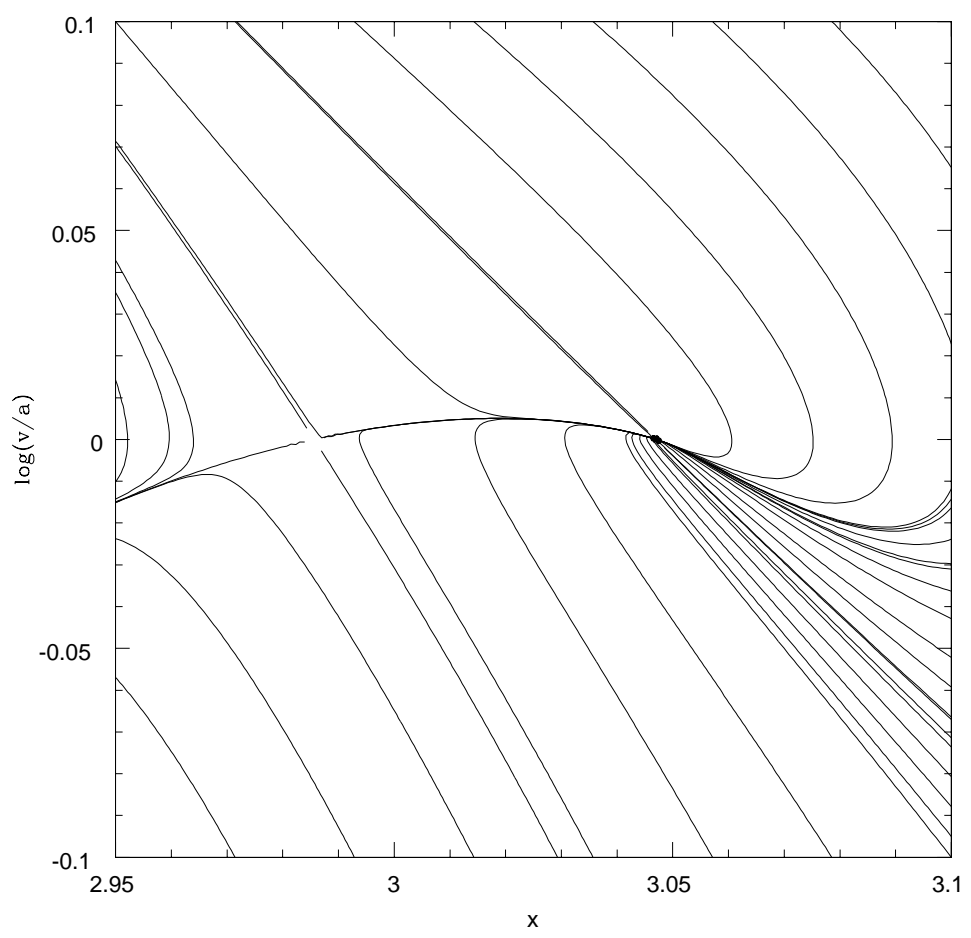

Fig. 10. - The vicinity of the critical points in the phase portrait of Fig.(9). 


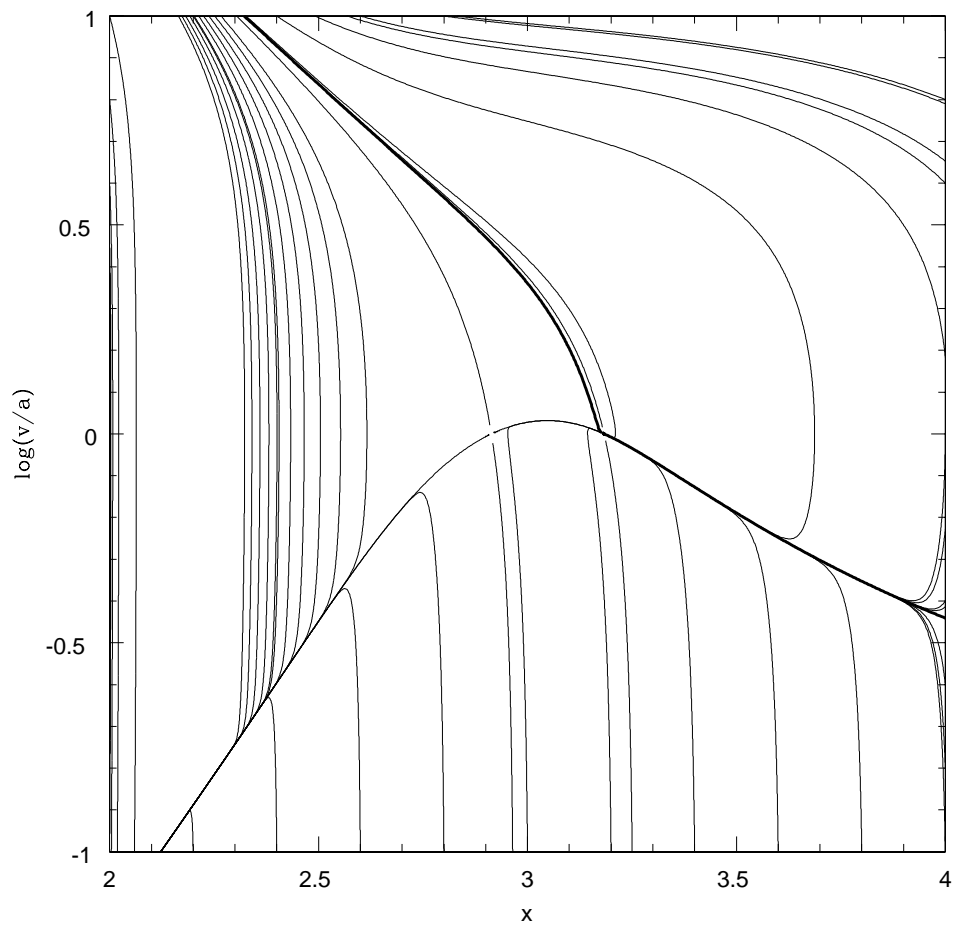

Fig. 11. - The Phase Portrait for $a=0.01, \alpha=0.3$ and $x_{c} \simeq 3.183$. The physical solution (thick curve) passes a critical point of the nodal type, in the slow direction, analytically.

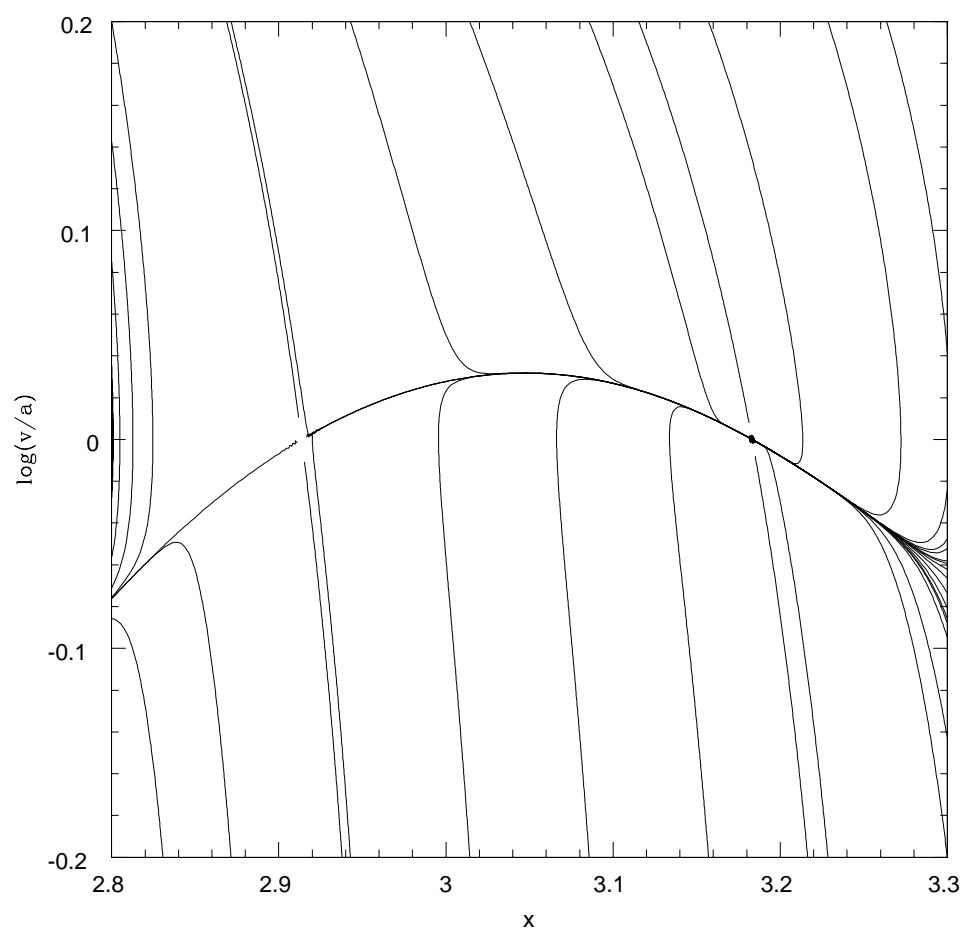

Fig. 12. - The vicinity of the critical points in the phase portrait of Fig.(11). 


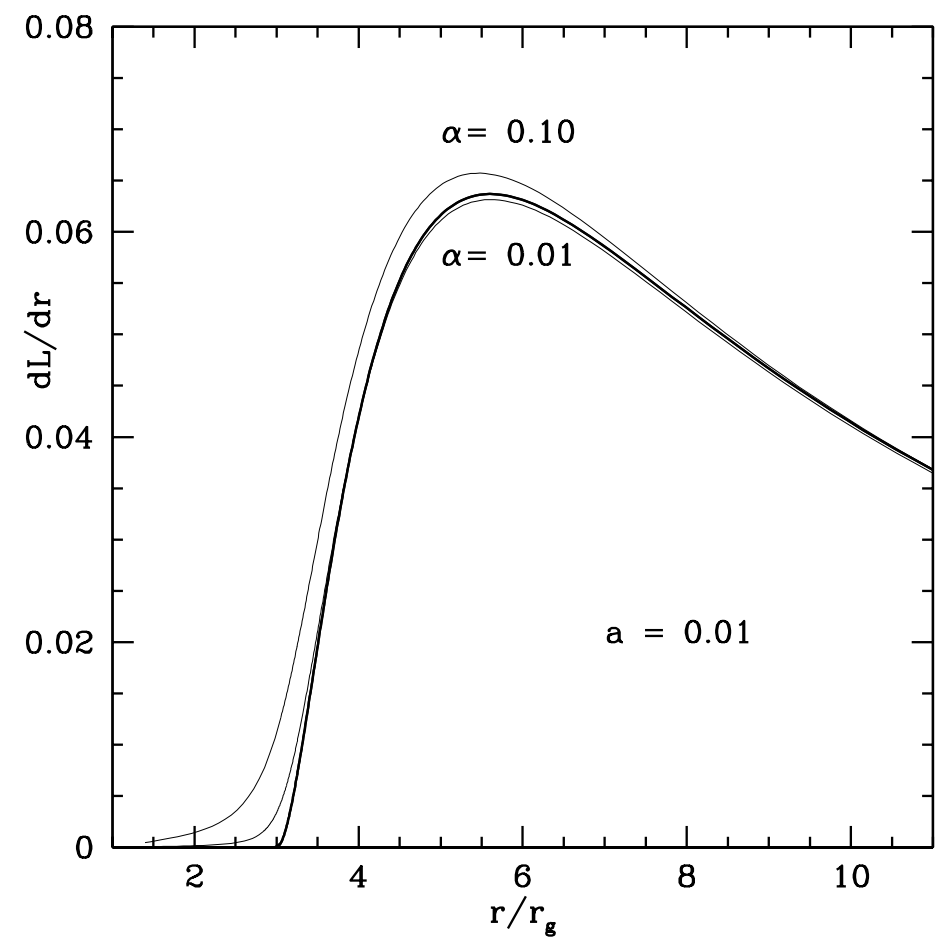

Fig. 13. - The energy generation rate in the classical model is shown with a thick solid line, and for our $a=0.01$ disk model with two thin lines, corresponding to $\alpha=0.01$ and $\alpha=0.1$, respectively. The disk luminosity is normalized to unity for the classical model. 


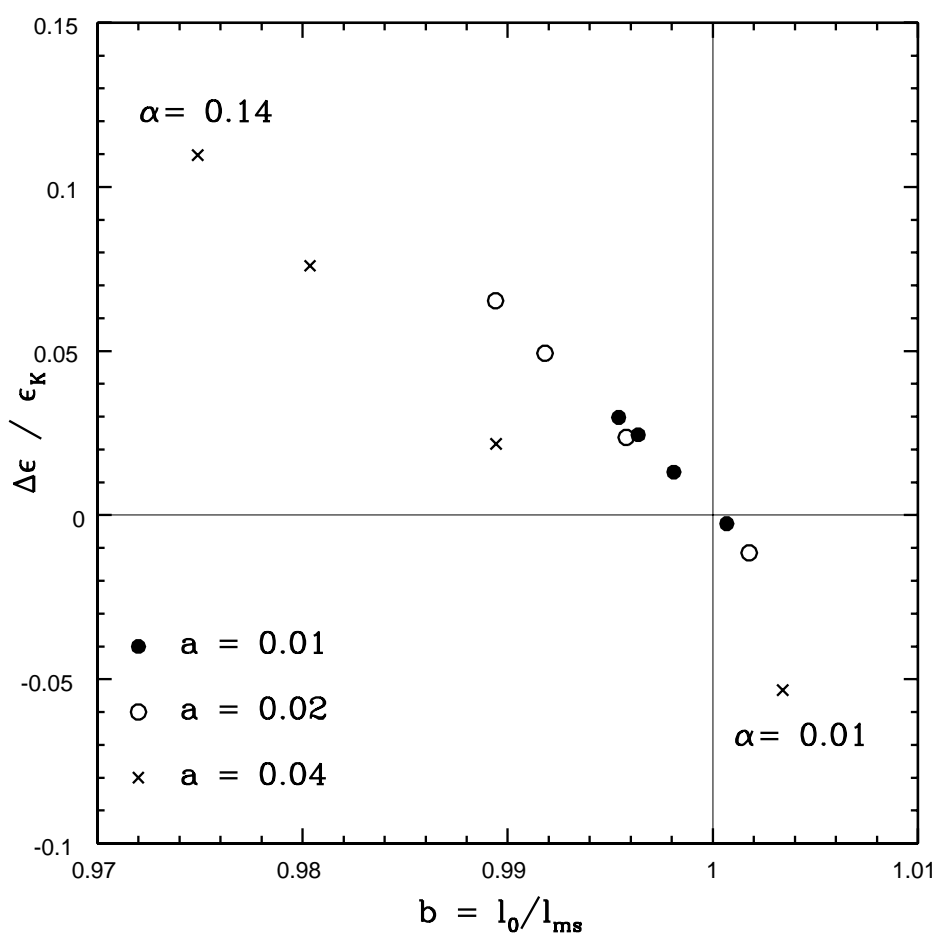

Fig. 14. - The relative change in the accretion efficiency for our disk models with constant effective speed of sound: $v_{s}=a c$ are shown for three values of $a(0.01,0.02,0.04)$ and for four values of the parameter $\alpha(0.01,0.05,0.10,0.14)$. For $\alpha=0.01$ the angular momentum constant $l_{0}$ is smaller than $l_{m s}$ and the accretion efficiency is reduced compared to the model with exactly zero torque at $r_{m s}$. The efficiency of accretion increases with increasing $\alpha$ and with increasing disk thickness, which is proportional to $a$. 